\title{
Second annotated list of type specimens of molluscs deposited in the Museu de Zoologia da Universidade de São Paulo, Brazil
}

\author{
Daniel C. CAVALLARI I,*, Ana Paula S. DORNELLAS ${ }^{2} \&$ Luiz Ricardo L. SIMONE ${ }^{3}$ \\ 1,2,3 Museu de Zoologia da Universidade de São Paulo, Cx. Postal 42494, 04218-970 São Paulo, SP, Brazil. \\ *Corresponding author: dccavallari@gmail.com \\ ${ }^{2}$ Email: dornellas.anapaula@gmail.com \\ ${ }^{3}$ Email: 1rsimone@usp.br \\ ${ }^{1}$ urn:Isid:zoobank.org:author:D0D70348-AF5B-417F-91BC-43DF9951D895 \\ ${ }^{2}$ urn:Isid:zoobank.org:author:B4162AEE-63BF-43D5-AABE-455AC51678BA \\ ${ }^{3}$ urn:lsid:zoobank.org:author:E66B5424-8F32-4710-B332-F35B9C8B84B0
}

\begin{abstract}
An alphabetical list of 352 type lots of molluscs housed in the Museu de Zoologia da Universidade de São Paulo is presented following the standards of the previous list by Dornellas \& Simone (2011), with a few adjustments. Important items listed herein include types of species described after the previous compilation, as well as recently acquired paratypes of Asian Pomatiopsidae and Diplommatinidae (Gastropoda) taxa described by Rolf A.M. Brandt (1960s), P. Temcharoen (1970s) and W.J.M. Maassen (2000s), all of which belonged to the private collection of Jens Hemmen, Wiesbaden, Germany. Relevant items also include types of recently described species coming from the FrenchBrazilian Marion Dufresne MD55 expedition, and other types deposited by researchers from Brazil and the world. A list of authors and photographs of specimens are also provided.
\end{abstract}

Keywords. Catalogue, Diplommatinidae, Pomatiopsidae, primary types, secondary types.

Cavallari D.C., Dornellas A.P.S. \& Simone L.R.L. 2016. Second annotated list of type specimens of molluscs deposited in the Museu de Zoologia da Universidade de São Paulo, Brazil. European Journal of Taxonomy 213: 1-59. http://dx.doi.org/10.5852/ejt.2016.213

\section{Introduction}

The malacological collection of the Museu de Zoologia da Universidade de São Paulo (MZSP), Brazil, is probably the largest in Latin America. Built through the continuous efforts of several researchers along the past centuries, it harbors over 122000 lots and over 1.2 million specimens of molluscs, including shells, soft parts and fossils.

Of the nearly 30000 lots incorporated into the MZSP collection after the previous compilation of type specimens (Dornellas \& Simone 2011), 352 are types. Some of the most important recently acquired items include numerous paratypes of Asian pomatiopsid taxa described by Rolf A.M. Brandt and P. Temcharoen in the late 1960s and early 1970s, as well as many diplommatinids described by W.J.M. 
Maassen during the past decade. These specimens belonged to the private collection of Jens Hemmen (1944-2012), Wiesbaden, Germany. Interested in malacology since his early student life in the 1960s, Hemmen became a well-known shell collector, dealer, and researcher. Along with his wife Christa, he built a large assemblage during his active years, having travelled and collected molluscs in several countries worldwide. He donated or sold specimens to numerous institutions, including more than 22000 specimens to the Academy of Natural Sciences of Philadelphia, USA, over 11000 specimens to the Wiesbaden Museum, and a few specimens to the Senckenberg Museum, Germany (Kittel et al. 2012). Still, a considerable part of his collection was sold to private collectors and dealers, and some of the specimens were subsequently acquired by the MZSP.

A large portion of the items listed herein was collected during the cruise of the R/V Marion Dufresne (MD55), Terres Australes et Antarctiques Françaises, a joint project of the Muséum national d'Histoire naturelle (MNHN, Paris, France) and the Universidade Santa Úrsula (USU, Rio de Janeiro, Brazil). This expedition took place during May and June 1987 and recovered a vast quantity of deep-water Mollusca off southeastern Brazil (Tavares 1999). Part of the MD55 specimens, which includes the items listed below, were kindly donated to the MZSP for study. Other than that, most of the new types stem from the efforts of Brazilian malacologists aimed at describing the local fauna, as well as donations from researchers worldwide.

In the continuous effort to publish the types of the MZSP's malacological collection, we provide here a second annotated list including all the items incorporated after the previous compilation (types from the previous list are not included in this work). A few items that were subsequently confirmed as types or inadvertently left out of the previous list are also discussed herein. We list primary and secondary types, possible paratypes, as well as any relevant topotypes. We provide colour photographs of older or non-illustrated taxa, references to the original descriptions, information on preservation, as well as any other relevant data on the specimens. We also take the opportunity to present a brief corrigendum to the previous list.

\section{Material and methods}

The list presented below follows the standards of Dornellas \& Simone (2011), with some modifications. The taxa are arranged in alphabetical order of specific epithets. After the specific epithet, the genus and author are given, followed by the year of description. The second line contains a citation to the original description, including a full range of pages and indications of the original figures when applicable. The remaining information is displayed according to the following template (modified after Dornellas \& Simone 2011):

Class, Family

Holotype: MZSP Number (Figure reference)

Paratype (number of $s p c$ ): MZSP Number (Figure reference, when applicable)

Paratype $^{2}$ (number of $s p c$ ): MZSP Number (Figure reference, when applicable)

Locality: Type locality; 1) Locality of paratype ${ }^{1}$ (when applicable); 2) Locality of paratype ${ }^{2}$ (when applicable).

Collector: Collector of holotype; 1) Collector of paratype ${ }^{1}$ (when applicable); 2) Collector of paratype ${ }^{2}$ (when applicable).

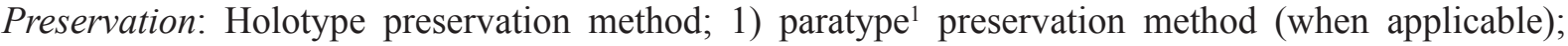
2) paratype ${ }^{2}$ preservation method (when applicable).

Remarks: Additional information on origin, previous collections, donations and taxonomy.

It should be noted that the superscripts for paratypes and topotypes do not correspond to any designation by the original author. Such information is mentioned among the remarks when applicable. Unless 
otherwise stated, paratype lots contain a single specimen and are organized in numerical order of catalogue numbers. Localities are given only after the list of type lots for each species, and individual localities for each lot are arranged in numerical order corresponding to their respective superscripts (e.g., 1) Brazil, Alagoas state; 2) Argentina, Buenos Aires; etc.). The systematic arrangement follows the World Register of Marine Species (WoRMS 2015) database for marine groups, and Bouchet et al. (2005) for the remaining taxa, with some modifications after Simone (2011).

\section{Institutional abbreviations}

INPA = Instituto Nacional de Pesquisas da Amazônia, Brazil

MNHN = Muséum national d'Histoire naturelle, France

MZSP = Museu de Zoologia da Universidade de São Paulo, Brazil

RMNH = Rijksmuseum van Natuurlijke Historie, Netherlands (currently Naturalis Biodiversity Center)

SEATO = South East Asian Treaty Organisation, Thailand

SMRL $=$ SEATO Medical Research Laboratory, Thailand

UNIRIO = Universidade Federal do Estado do Rio de Janeiro, Brazil

USU = Universidade Santa Úrsula, Brazil

\section{Research project abbreviations}

PADCT = "Importância e Caracterização da Quebra de Plataforma Continental para Recursos Vivos e Não Vivos", Brazil.

REVIZEE = "Programa de Avaliação do Potencial Sustentável de Recursos Vivos da Zona Econômica Exclusiva", Brazil.

\section{Results}

\section{Annotated list of type specimens}

abundans, Diplommatina Maassen, 2002

Diplommatina abundans Maassen, 2002: 165 (figs 1-2).

Gastropoda, Diplommatinidae

Probable paratype: MZSP 95465 (Fig. 1E-F).

Locality: Indonesia, West Sumatra, SE of Bukit Tingi, $3 \mathrm{~km} N$ of Lintau Village, near the entrance of Gua Pangian (type locality), 1996.

Preservation: Dry.

Remarks: Former Jens Hemmen Collection. Data on specimen's label compare fittingly with a 100 -specimen paratype series originally deposited in Maassen's reference collection.

\section{adami, Paraprososthenia Brandt, 1970}

Paraprososthenia adami Brandt, 1970: 189-190 (pl. 13, fig. 7 + text fig. 7).

Gastropoda, Pomatiopsidae

Probable paratype: MZSP 95923.

Locality: Cambodia, Mekong River at Sambor (type locality), N of Kratie, 6 Apr. 1969.

Preservation: Dry.

Remarks: Former Jens Hemmen Collection. Measurements and data on the specimen's label correspond to the 70-specimen paratype lot originally catalogued as SMRL 16302. 


\section{ahiparana, Turritella Powell, 1927}

Turritella (Zeacolpus) ahiparana Powell, 1927: 297 (pl. 34, fig. 4).

Gastropoda, Turritellidae

Probable paratype: MZSP 101757 (Fig. 5C-D).

Locality: New Zealand, Northland, Ahipara, $42 \mathrm{~m}$ depth.

Preservation: Dry.

Remarks: The holotype, as well as several paratypes of $Z$. ahiparana, were deposited in Powell's private collection, and only the former was figured and measured. The probable paratype listed above belonged to the private shell collector Ralph Humes, who had a large private assemblage in the late 1950s. His collection contained 850 lots, including types. The Ralph Humes Collection label bears a handwritten paratype status annotation. This species was recombined as Stiracolpus ahiparanus by Beu (2011).

\section{akangus, Dolichupis Simone \& Cunha, 2012}

Dolichupis akangus Simone \& Cunha, 2012: 754-756 (fig. 4A-D).

Gastropoda, Triviidae

Paratypes (2 spc): MZSP 104027.

Paratypes $^{1}(2$ spc): MZSP 104028.

Paratypes $^{2}$ (4 spc): MZSP 104029.

Localities: Brazil, Espírito Santo, off Regência, 1940" S, 3748' W, MD55 sta. CB77, 790-940 m depth, 27 May 1987; 1) off Itaúnas, Abrolhos Slope, 1856' S, 3752' W, MD55 sta. DC82, 85-105 m, 28 May 1987; 2) off Itaúnas, 1859'S, 37²48' W, 607-620 m, 27 May 1987.

Collectors: P. Bouchet, J.H. Leal and B. Métivier.

Preservation: Dry.

Remarks: Former MNHN, Paris.

\section{alagoensis, Terebra Lima, Tenório \& Barros, 2007}

Terebra alagoensis Lima, Tenório \& Barros, 2007: 66 (figs 13-17).

Gastropoda, Terebridae

Holotype: MZSP 84238.

Locality: Brazil, off Alagoas, continental slope, $10^{\circ} 05^{\prime} 57^{\prime \prime}$ S, 3546'24" W, D-29, 720 m depth, 28 Oct. 2000.

Collector: R/V Natureza.

Preservation: Dry.

alaris, Cranopsis Simone \& Cunha, 2014

Cranopsis alaris Simone \& Cunha, 2014: 448-449 (figs 66-75).

Gastropoda, Fissurellidae

Paratypes (2 spc): MZSP 105512.

Locality: Brazil, Espírito Santo, Abrolhos Slope, off Itaúnas, 1859' S, 3750' W, 295 m depth, MD55 sta. DC75 (type locality), 27 May 1987.

Collectors: P. Bouchet, J.H. Leal and B. Métivier.

Preservation: Dry.

Remarks: Former MNHN, Paris.

alvesi, Gerdiella Lima, Barros \& Petit, 2007

Gerdiella alvesi Lima, Barros \& Petit, 2007: 100-102 (figs 1-14). 


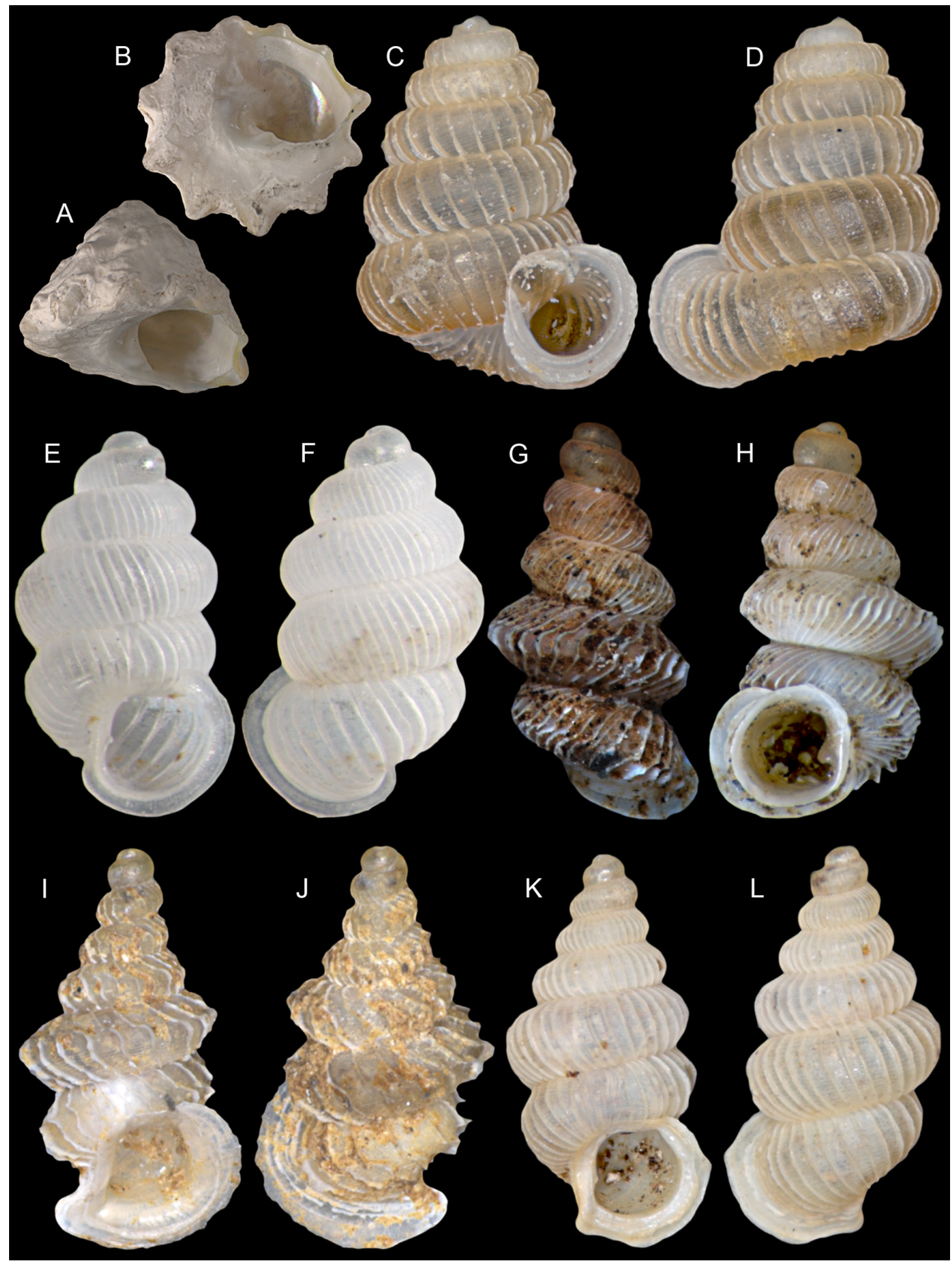

Fig. 1. A-B. Astraea danieli Alf \& Kreipl, 2006, paratype MZSP 95919, L = $11.5 \mathrm{~mm}, \mathrm{~W}=14.5 \mathrm{~mm}$. C-D. Arinia panhai Maassen, 2001, paratype MZSP 95493, L $=2.58 \mathrm{~mm}, \mathrm{~W}=2 \mathrm{~mm}$. E-F. Diplommatina abundans Maassen, 2002, paratype MZSP 95465, L $=1.34 \mathrm{~mm}, \mathrm{~W}=0.7 \mathrm{~mm}$. G-H. Diplommatina carinaspinosa Maassen, 2002, paratype MZSP 95941, L $=1.83 \mathrm{~mm}, \mathrm{~W}=0.83 \mathrm{~mm}$. I-J. Diplommatina supralamellata Maassen, 2007, probable paratype MZSP 95498, L $=3.37 \mathrm{~mm}, \mathrm{~W}=1.75 \mathrm{~mm}$. K-L. Diplommatina wilhelminae Maassen, 2002, probable paratype MZSP 95462, L $=2.87 \mathrm{~mm}$, $\mathrm{W}=1.37 \mathrm{~mm}$. 
Gastropoda, Cancellariidae

Holotype: MZSP 78932.

Paratypes (4 spc): MZSP 78933.

Locality: Northeastern Brazil, off Pernambuco, muddy bottom, 0846.5' S, 34²44.5' W, 690 m depth (type locality), 18 Nov. 2000.

Preservation: Dry.

Remarks: The holotype and one of the four MZSP paratypes were figured in the original description (Lima et al. 2007a: respectively figs 1-5 and figs 12-13).

\section{amandus, Megalobulimus Simone, 2012}

Megalobulimus amandus Simone, 2012b: 436-438 (figs 22-26).

Gastropoda, Strophocheilidae

Holotype: MZSP 103917.

Paratypes (10 spc): MZSP 103918.

Paratypes (3 spc): MZSP 103919.

Locality: Brazil, Bahia, Santa Maria da Vitória, $\sim 13^{\circ} 24^{\prime} \mathrm{S}, 4^{\circ} 12^{\prime} \mathrm{W}, \sim 460 \mathrm{~m}$ altitude (type locality), Jan. 2012.

Collector: J. Coltro.

Preservation: Dry.

\section{amphissa, Turricostellaria Simone \& Cunha, 2012}

Turricostellaria amphissa Simone \& Cunha, 2012: 758-759 (fig. 5A-D).

Gastropoda, Costellariidae

Paratypes (2 spc): MZSP 102691.

Locality: Brazil, Espírito Santo, off Itaúnas, Abrolhos Slope, 1859' S, 3750' W, MD55 sta. DC75, 295 m depth, 27 May 1987.

Collectors: P. Bouchet, J.H. Leal and B. Métivier.

Preservation: Dry.

Remarks: Former MNHN, Paris.

\section{analuciae, Bullata Souza \& Coovert, 2001}

Bullata analuciae de Souza \& Coovert, 2001: 5-6 (figs 2, 8, 11).

Gastropoda, Marginellidae

Paratype: MZSP 28243 (Fig. 5G-H).

Locality: Brazil, Espírito Santo, Vitória, 2020' S, 4000' W, 60-70 m depth (type locality), Apr. 1996.

Collector: Shrimp fishing boat.

Preservation: Dry.

\section{angrensis, Eurytellina Marques \& Simone, 2014}

Eurytellina angrensis Marques \& Simone, 2014: 40-46 (figs 1-35).

Bivalvia, Tellinidae

Holotype: MZSP 21720.

Paratype $^{1}$ : MZSP 41519.

Paratype $^{2}$ : MZSP 58933.

Paratype $^{3}$ : MZSP 84044.

Paratype $^{4}$ : MZSP 108179.

Paratype $^{5}$ : MZSP 108182. 
Localities: Brazil, Rio de Janeiro, Ilha Grande Bay, along the coast of Angra dos Reis, $23^{\circ} 06^{\prime} \mathrm{S}, 44^{\circ} 36^{\prime} \mathrm{W}$;

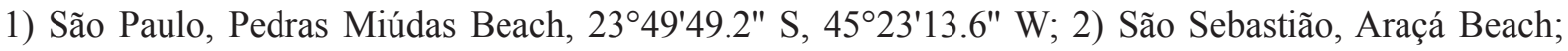
3) Ilhabela, Barreiro Beach, $23^{\circ} 48^{\prime} 57.65^{\prime \prime} \mathrm{S}, 4^{\circ} 22^{\prime} 19.34^{\prime \prime}$ W; 4) Santa Catarina, Porto Belo; 5) Rio de Janeiro state, Angra dos Reis, Enseada das Palmas, Ilha Grande City.

Preservation: holotype $70 \%$ ethanol; paratypes are preserved dry.

\section{aninga, Cornisepta Simone \& Cunha, 2014}

Cornisepta aninga Simone \& Cunha, 2014: 441-442 (figs 17-27).

Gastropoda, Fissurellidae

Paratypes (10 spc): MZSP 102942.

Locality: Brazil, Espírito Santo, Abrolhos Slope, off Itaúnas, 1859' S, 3750' W, 295 m depth, MD55 sta. DC75 (type locality), 27 May 1987.

Collectors: P. Bouchet, J.H. Leal and B. Métivier.

Preservation: Dry.

Remarks: Former MNHN, Paris.

\section{angularis, Corinnaeturris Figueira \& Absalão, 2010}

Corinnaeturris angularis Figueira \& Absalão, 2010a: 739 (fig. 2G-J).

Gastropoda, Clathurellidae

Paratype: MZSP 95878.

Locality: Brazil, Rio de Janeiro, Campos Basin, 2204'32" S, 3954'11" W, sta. OPII\#49, 722 m, 30 Jun. 2003.

Collector: R/V Astro Garoupa.

Preservation: Dry.

\section{aperta, Lithoglyphopsis Temcharoen, 1971}

Lithoglyphopsis aperta Temcharoen, 1971: 103-104 (pl. 7, fig. 14 + text fig. 12).

Gastropoda, Pomatiopsidae

Probable paratype: MZSP 95942 (Fig. 5A-B).

Locality: Laos, Mekong River, Khong Island, near Wat, 28 Feb. 1969.

Preservation: Dry.

Remarks: Former Jens Hemmen Collection. Measurements and data on the specimen's label correspond to a 30 -specimen paratype lot originally catalogued as SMRL 16336. This species was recombined as Neotricula aperta by Davis et al. (1986).

\section{apiculus, Microlinices Simone, 2014}

Microlinices apiculus Simone, 2014: 580 (fig. 8C-G).

Gastropoda, Naticidae

Paratypes (6 spc): MZSP 105146.

Locality: Brazil, Espírito Santo, off Conceição da Barra, Abrolhos Slope, 1859' S, 3750' W, MD55 sta. CB76, 637 m (type locality), 27 May 1987.

Collectors: P. Bouchet, J.H. Leal and B. Métivier.

Preservation: Dry.

Remarks: Former MNHN, Paris. 


\section{apostrema, Cranopsis Simone \& Cunha, 2014}

Cranopsis apostrema Simone \& Cunha, 2014: 448 (figs 56-65).

Gastropoda, Fissurellidae

Paratypes (10 spc): MZSP 105509.

Locality: Brazil, Espírito Santo, off Itaúnas, Abrolhos Slope, 1859' S, 3750' W, 295 m (type locality), 27 May 1987.

Collectors: P. Bouchet, J.H. Leal and B. Métivier.

Preservation: Dry.

\section{apyrahi, Turricostellaria Simone \& Cunha, 2012}

Turricostellaria apyrahi Simone \& Cunha, 2012: 759 (fig. 5L-R).

Gastropoda, Costellariidae

Paratype: MZSP 102862.

Paratype: MZSP 102863.

Locality: Brazil, Espírito Santo, off Itaúnas, Abrolhos Slope, 1901' S, 3747’ W, MD55 sta. CB79, 1500-1575 m depth, 28 May 1987.

Collectors: P. Bouchet, J.H. Leal and B. Métivier.

Preservation: Dry.

Remarks: Former MNHN, Paris.

\section{aquitanus, Onustus Simone \& Cunha, 2012}

Onustus aquitanus Simone \& Cunha, 2012: 750-751 (fig. 2).

Gastropoda, Xenophoridae

Paratype: MZSP 104032.

Locality: Brazil, Espírito Santo, off Conceição da Barra, Abrolhos Slope, 1859' S, 3750' W, MD55 sta. DC73, $637 \mathrm{~m}$ depth, 27 May 1987.

Collectors: P. Bouchet, J.H. Leal and B. Métivier.

Preservation: Dry.

Remarks: Former MNHN, Paris.

\section{arariboia, Yoldiella Benaim \& Absalão, 2011}

Yoldiella arariboia Benaim \& Absalão, 2011: 521-523 (fig. 6).

Bivalvia, Yoldiidae

Paratypes (6 spc): MZSP 93358.

Locality: Brazil, Rio de Janeiro, Campos Basin, sta. 84, 22²6'28" S, 3958'53.3" W, 1050 m depth, 2003.

Collector: R/V Astro Garoupa.

Preservation: Dry.

\section{arawak, Jaspidiconus Petuch \& Myers, 2014}

Jaspidiconus arawak Petuch \& Myers, 2014a: 40-41 (fig. 2J-K).

Gastropoda, Conidae

Holotype: MZSP 116149.

Locality: Lesser Antilles Archipelago, Grenadines (Dependency of Grenada), Petit Martinique Island, 3 m depth.

Preservation: Dry.

Remarks: This species was recombined as Conasprella arawak by Puillandre et al. (2015). 


\section{arrepiata, Cornisepta Simone \& Cunha, 2014}

Cornisepta arrepiata Simone \& Cunha, 2014: 441 (figs 6-16).

Gastropoda, Fissurellidae

Paratypes (9 spc): MZSP 102943.

Paratype $^{1}$ : MZSP 102944.

Paratype $^{2}$ : MZSP 102945.

Localities: Brazil, Espírito Santo, Abrolhos Slope, off Itaúnas, 1859' S, 3750' W, 295 m depth, MD55 sta. DC75 (type locality); 1) off Regência, 1940' S, 37048' W, 790-940 m, MD55 sta. CB77; 2) off Itaúnas, 1859' S, 37²48' W, 607-620 m, MD55 sta. DC73; all collected on 27 May 1987.

Collectors: P. Bouchet, J.H. Leal and B. Métivier.

Preservation: Dry.

assu, Terebra Simone \& Cunha, 2012

Terebra assu Simone \& Cunha, 2012: 772-774 (figs 10A-E).

Gastropoda, Terebridae

Paratype: MZSP 102598.

Locality: Brazil, Espírito Santo, off Conceição da Barra, Abrolhos Slope, $18^{\circ} 59^{\prime}$ S, 3750' W, MD55 sta. DC75, 295 m depth, 27 May 1987.

Collectors: P. Bouchet, J.H. Leal and B. Métivier.

Preservation: Dry.

Remarks: Former MNHN, Paris.

\section{astirakiensis, Orculella Gittenberger \& Hausdorf, 2004}

Orculella astirakiensis Gittenberger \& Hausdorf, 2004: 104-105 (figs 21, 46).

Gastropoda, Orculidae

Paratype: MZSP 95489 (Fig. 7C-D).

Locality: Greece, Crete, km 18 of old road Iraklio, 28 Oct. 1990.

Preservation: Dry.

Remarks: Former Jens Hemmen Collection. The specimen listed above is a remainder of a 17 -specimen paratype lot deposited in Hemmen's collection by Gittenberger \& Hausdorf (2004).

asymmetrica, Ervilia Marques \& Simone, 2011

Ervilia asymmetrica Marques \& Simone, 2011: 652-653 (figs 1-7).

Bivalvia, Semelidae

Holotype (left valve): MZSP 96885.

Paratype (right valve): MZSP 96886.

Paratype (right valve): MZSP 96887.

Paratype (left valve): MZSP 96888.

Locality: Brazil, Pará, Amazon River mouth, 85 km off Ilha Mexiana, 0²7'23.28" N, 48¹3'56.84" W, PIATAM Oceano (type locality), 10 Mar. 2009.

Collector: F.N. Santos.

Preservation: Dry.

atiaia, Manganesepta Simone \& Cunha, 2014

Manganesepta atiaia Simone \& Cunha, 2014: 456-457 (figs 103-108).

Gastropoda, Fissurellidae 
Paratypes (2 spc): MZSP 102941.

Paratype $^{1}$ : MZSP 102946.

Localities: Brazil, Espírito Santo, Abrolhos Slope, off Itaúnas, $19^{\circ} 00^{\prime} \mathrm{S}, 37^{\circ} 49^{\prime} \mathrm{W}, 950-1050 \mathrm{~m}$ depth, MD55 sta. DC77 (type locality); 1) off Itaúnas, $1^{\circ} 01^{\prime} \mathrm{S}, 37^{\circ} 47^{\prime} \mathrm{W}, 1500-1575$ m, MD55 sta. CB79; all collected on 27 May 1987.

Collectors: P. Bouchet, J.H. Leal and B. Métivier.

Preservation: Dry.

\section{bavayi, Pachydrobia Brandt, 1970}

Pachydrobia bavayi Brandt, 1970: 192-193 (pl. 13, fig. $11+$ text fig. 11).

Gastropoda, Pomatiopsidae

Probable paratype: MZSP 95911 (Fig. 2E-F).

Locality: Cambodia, Mekong River at Kratie (type locality), 3 Apr. 1969.

Preservation: Dry.

Remarks: Former Jens Hemmen Collection. Measurements and data on the specimen's label correspond to the lot originally registered under code SMRL 16285. The specimen listed above is a possible remainder of a 200 -specimen paratype lot coming from the type locality.

\section{benthovus, Microlinices Simone, 2014}

Microlinices benthovus Simone, 2014: 575-578 (figs 6A-J, 7A-H, 11A-C).

Gastropoda, Naticidae

Paratypes (22 spc): MZSP 105269.

Paratypes $^{1}(15$ spc): MZSP 105270.

Paratypes $^{2}$ (16 spc): MZSP 105271.

Paratypes $^{3}$ (7 spc): MZSP 105272.

Localities: Brazil, Espírito Santo, off Itaúnas, Abrolhos Slope, 1859' S, 3750' W, MD55 sta. DC73,

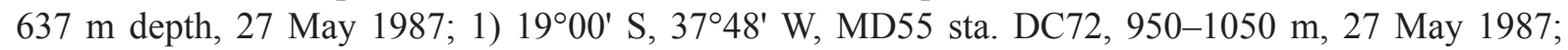
2) off Regência, 1940' S, 3748' W, MD55 sta. CB77, 790-940 m depth, 27 May 1987; 3) off Itaúnas, Abrolhos Slope, $1^{\circ} 01^{\prime}$ S, 3747' W, MD55 sta. CB79, 1500-1575 m depth, 28 May 1987.

Collectors: P. Bouchet, J.H. Leal and B. Métivier.

Preservation: Dry.

Remarks: Former MNHN, Paris. The catalogue number MZSP 105250 is mentioned twice in Simone's (2014) paper, among the paratypes of M. ibitingus Simone, 2014 and M. benthovus. This duplicity was a mistake by the author: the latter is an erroneous designation and should be disregarded. The only valid paratype lots for M. benthovus are the ones shown above.

\section{berschaueri, Jaspidiconus Petuch \& Myers, 2014}

Jaspidiconus berschaueri Petuch \& Myers, 2014a: 41-42 (fig. 2D-F).

Gastropoda, Conidae

Holotype: MZSP 116150.

Locality: Lesser Antilles, Sint Maarten Island, Great Bay, Philipsburg, along the shore.

Collector: D. Berschauer.

Preservation: Dry.

Remarks: This species was recombined as Conasprella berschaueri by Puillandre et al. (2015).

\section{biocellatus, Pustulatirus Lyons \& Snyder, 2013}

Pustulatirus biocellatus Lyons \& Snyder, 2013: 48 -49 (figs 46-51). 


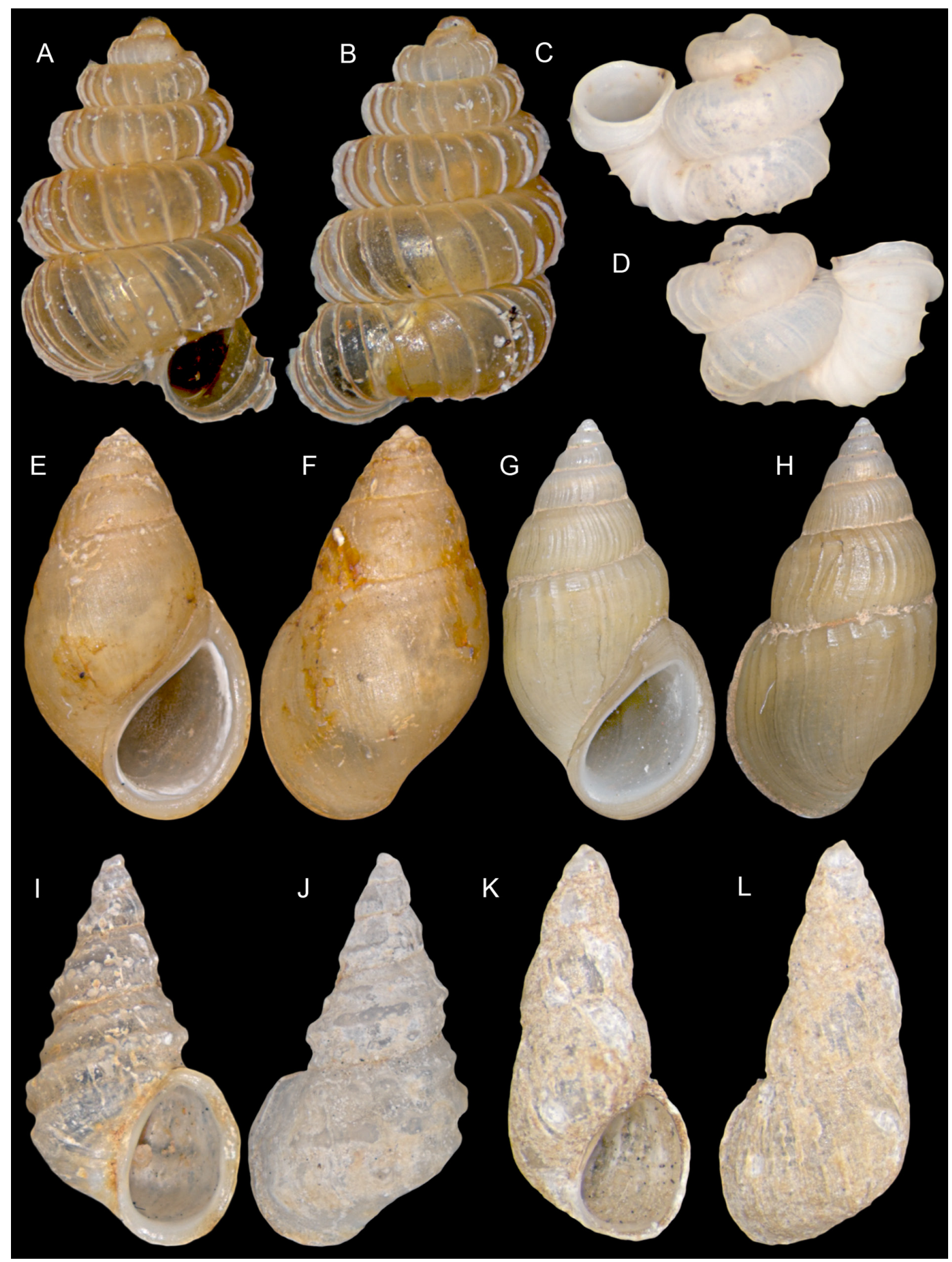

Fig. 2. A-B. Opisthostoma christae Maassen, 2001, paratype MZSP 95472, L = 2.55 mm, W $=1.55 \mathrm{~mm}$. C-D. Opisthostoma secretum Maassen, 2002, probable paratype MZSP 95917, L $=0.9 \mathrm{~mm}$, $\mathrm{W}=1.28 \mathrm{~mm}$. E-F. Pachydrobia bavayi Brandt, 1970, probable paratype MZSP 95911, L $=6.8 \mathrm{~mm}$, $\mathrm{W}=4 \mathrm{~mm}$. G-H. Pachydrobia crooki Brandt, 1968, probable paratype MZSP 95469, L $=12.8 \mathrm{~mm}$, $\mathrm{W}=6.8 \mathrm{~mm}$. I-J. Paraprososthenia brandti Temcharoen, 1971, probable paratype MZSP 95946, $\mathrm{L}=5.25 \mathrm{~mm}, \mathrm{~W}=3 \mathrm{~mm}$. K-L. Paraprososthenia fischerpiettei Brandt, 1970, probable paratype MZSP 95496, $\mathrm{L}=4.72 \mathrm{~mm}, \mathrm{~W}=2.2 \mathrm{~mm}$. 
Gastropoda, Fasciolariidae

Holotype: MZSP 108767.

Locality: Brazil, Rio Grande do Norte, off northern Natal, on sandy bottom under rocks at 10-15 m depths.

Collectors: Collected by local people.

Preservation: Dry.

Remarks: Former Bill Lyons Collection, Florida, USA.

\section{boholensis, Strombus kleckhamae Mühlhäusser, 1981}

Strombus (Dolomena) kleckhamae boholensis Mühlhäusser, 1981: 319 (figs 1-4).

Gastropoda, Strombidae

Paratype: MZSP 95952.

Locality: Bohol Island, Philippines.

Preservation: Dry.

Remarks: Former Jens Hemmen Collection. The specimen listed above was referred to as "Paratypus 2" in the original description, and figured on page 321 (fig. 3). This species was recombined as Barneystrombus boholensis by Blackwood (2009).

\section{brachyplax, Hanleya Jardim \& Simone, 2010}

Hanleya brachyplax Jardim \& Simone, 2010b: 14.

Polyplacophora, Hanleyidae

Holotype: MZSP 87263.

Paratype $^{1}$ : MZSP 87264.

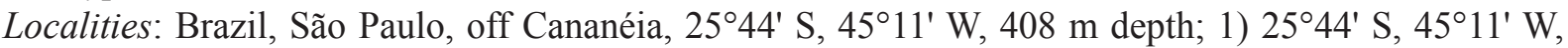
$400 \mathrm{~m}$ depth, 18 Apr. 2002.

Collectors: REVIZEE Score Sul project members.

Preservation: $70 \%$ ethanol; valves are preserved dry.

Remarks: Simone \& Jardim published a description of this species in Rios (2009). Nevertheless, it did not fulfill the requirements of ICZN articles 16.1 (the species' name must be explicitly indicated as intentionally new) and 16.4 (fixation of name-bearing types must be explicit). In the following year, Jardim \& Simone (2010a) published a new description with detailed figures and a holotype designation. However, since the new name was not explicitly and intentionally indicated, the requirements of ICZN article 16.1 were still not met in that paper. To correct this, the name Hanleya brachyplax was formally introduced in a subsequent note by Jardim \& Simone (2010b), which is the only valid description.

\section{brandti, Paraprososthenia Temcharoen, 1971}

Paraprososthenia brandti Temcharoen, 1971: 94 (pl. 6, fig. 3 + text fig. 3).

Gastropoda, Pomatiopsidae

Probable paratype: MZSP 95946 (Fig. 2I-J).

Locality: Laos, Sedone River at Khong Sedone, N of Pakse in Laos (type locality), 23 Feb. 1969.

Preservation: Dry.

Remarks: Former Jens Hemmen Collection. Measurements and data on the specimen's label compare fittingly with two paratype lots mentioned in the original description: SMRL 16109 and a 20-specimen lot originally deposited in the author's reference collection.

\section{breweri, Plekocheilus Breure \& Schlögl, 2010}

Plekocheilus (Eurytus) breweri Breure \& Schlögl, 2010: 57-58 (figs 2D-2G, 3C-D, 4B-4F). 
Gastropoda, Orthalicidae

Paratypes (2 spc): MZSP 92471.

Locality: Venezuela, Bolívar, Chimantá Massif, Curí-tepui, 05¹6'44.9" N, 6200'56.35" W, 2405 m depth (type locality), Feb. 2009.

Collector: J. Schögl.

Preservation: Dry.

\section{cambodiensis, Hydrorissoia Brandt, 1970}

Hydrorissoia cambodiensis Brandt, 1970: 186 (pl. 13, fig. 3 + text fig. 3).

Gastropoda, Pomatiopsidae

Probable paratype: MZSP 95912 (Fig. 3A-B).

Locality: Mekong at Sambor (type locality), 6 Apr. 1969.

Preservation: Dry.

Remarks: Former Jens Hemmen Collection. Measurements and data on the specimen's label correspond to the lot originally catalogued as SMRL 16296. The specimen listed above is a possible remainder of a 200 -specimen paratype lot coming from the type locality. This species was recombined as Halewisia cambodiensis by Davis (1979).

\section{canopa, Cranopsis Simone \& Cunha, 2014}

Cranopsis canopa Simone \& Cunha, 2014: 449-450 (figs 76-83).

Gastropoda, Fissurellidae

Holotype: MZSP 107718.

Paratypes (24 spc): MZSP 90281.

Paratypes (11 spc): MZSP 94183.

Locality: Brazil, Ceará, off Fortaleza, Canopus Bank, $2^{\circ} 12^{\prime} 43^{\prime \prime}$ S, 38 $38^{\circ} 18^{\prime} 52^{\prime \prime}$ W, 250 m depth (type locality), Aug. 2005.

Collector: J. Coltro.

Preservation: Dry.

\section{capivara, Cyclodontina Simone \& Casati, 2013}

Cyclodontina capivara Simone \& Casati, 2013: 149-151 (figs 16-22).

Gastropoda, Odontostomidae

Holotype: MZSP 112448.

Paratypes $^{1}$ (19 spc): MZSP 111837.

Paratypes $^{2}$ (18 spc): MZSP 111838.

Paratypes $^{3}$ (9 spc): MZSP 111845.

Localities: Brazil, Piauí, Serra da Capivara, Coronel José Dias Municipality, close to Sítio do Mocó town, Toca de Cima dos Pilão Cave, $8^{\circ} 51^{\prime} 47.10^{\prime \prime} \mathrm{S}, 42^{\circ} 33^{\prime} 26.96^{\prime \prime} \mathrm{W}$; 1-2) same, sta. P1; all collected in 2012; 3) from type locality.

Collector: R. Casati.

Preservation: Dry.

Remarks: Specimens in the lot MZSP 111835 were listed as paratypes of Rhinus gilbertus by Simone \& Casati (2013, original description), but are in fact topotypes of C. capivara, which is described in the same paper. The lot MZSP 111845 contains 9 shells and not 8 as reported in the original description of $C$. capivara. Simone \& Casati (2013) simultaneously reported the catalogue number MZSP 111845 among the paratypes of both C. capivara and Rhinus gilbertus. This catalogue number does not contain paratype specimens of $R$. gilbertus: this information is incorrect in the original description and should be disregarded. 


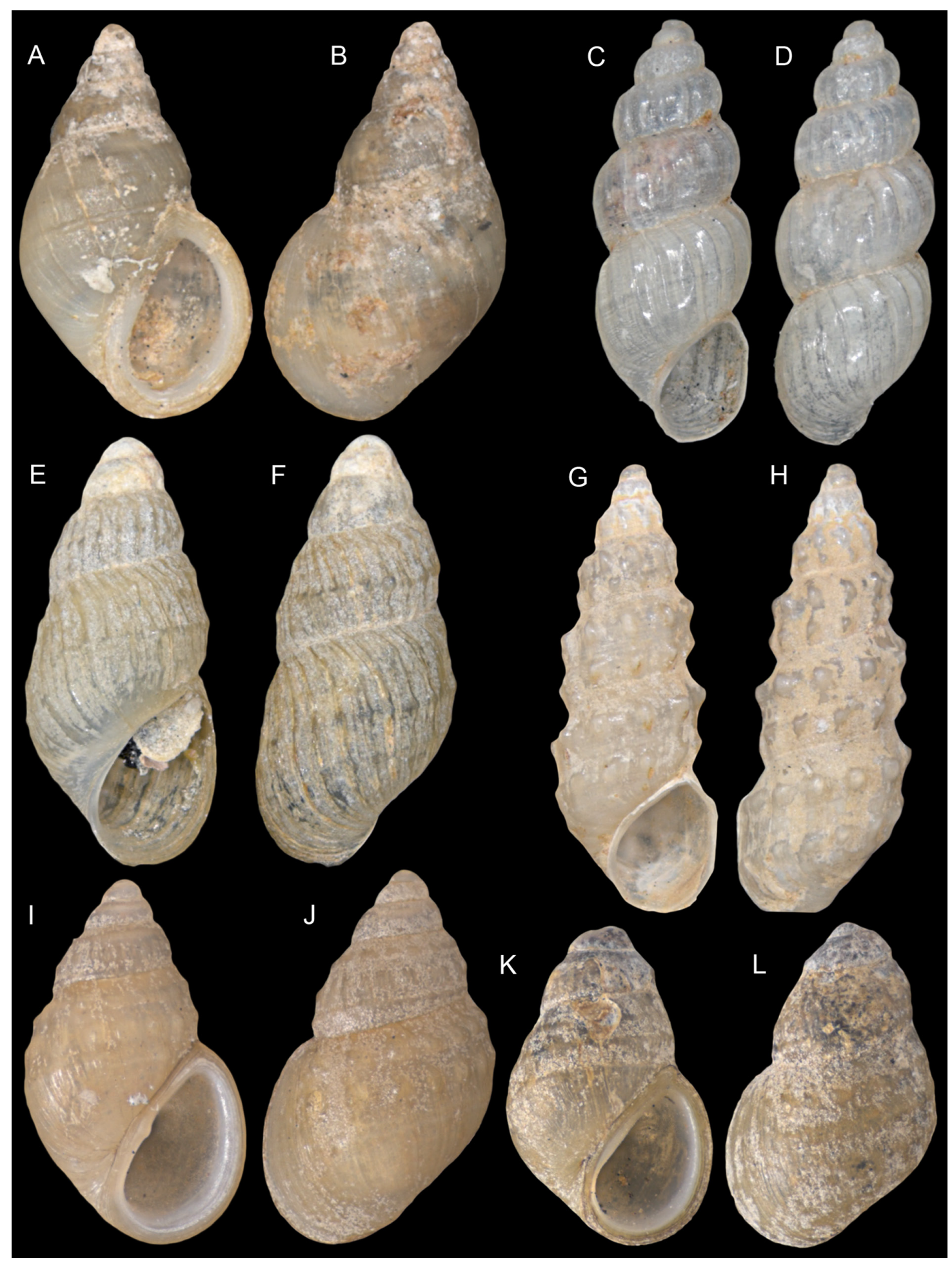

Fig. 3. A-B. Hydrorissoia cambodiensis Brandt, 1970, probable paratype MZSP 95912, L = $3.9 \mathrm{~mm}$, $\mathrm{W}=2.3 \mathrm{~mm}$. C-D. Hubendickia cylindrica Brandt, 1974, probable paratype MZSP 95944, L = $4.5 \mathrm{~mm}$, $\mathrm{W}=1.8 \mathrm{~mm}$. E-F. Hubendickia rolfbrandti Temcharoen, 1971, probable paratype MZSP 95948, $\mathrm{L}=4.1 \mathrm{~mm}, \mathrm{~W}=2 \mathrm{~mm}$. G-H. Paraprososthenia schuetti Brandt, 1968, probable paratype MZSP 95487, $\mathrm{L}=6.2 \mathrm{~mm}, \mathrm{~W}=2.1 \mathrm{~mm}$. I-J. Hydrorissoia hospitalis Brandt, 1968, probable paratype MZSP 95949, $\mathrm{L}=3.2 \mathrm{~mm}, \mathrm{~W}=1.9 \mathrm{~mm}$. K-L. Hydrorissoia munensis Brandt, 1968, probable paratype MZSP 95929, $\mathrm{L}=2.76 \mathrm{~mm}, \mathrm{~W}=1.74 \mathrm{~mm}$. 
Anna capixaba Coltro \& Dornellas, 2013: 97-99 (figs 1-6, 9-10).

Gastropoda, Buccinidae

Holotype: MZSP 101385.

Paratype: MZSP 101386.

Locality: Brazil, Espírito Santo, off Conceição da Barra, 18³6' S, 39³2' W, 40-50 m depths, Dec. 2009. Collector: J. Coltro.

Preservation: Dry.

carinaspinosa, Diplommatina Maassen, 2002

Diplommatina carinaspinosa Maassen, 2002: 167 (figs 5-7).

Gastropoda, Diplommatinidae

Probable paratype: MZSP 95941 (Fig. 1G-H).

Locality: Indonesia, West Sumatra, SE of Bukittinggi, $3 \mathrm{~km} \mathrm{~N}$ of village Lintau, near entrance of Cave Gua Pangian, 00²8'19.5" S, 100²5'11.7" E (type locality), Jul. 1996.

Collector: W.J.M. Maassen.

Preservation: Dry.

Remarks: Former Jens Hemmen Collection. Data on the specimen's label and measurements compare fittingly with a large paratype series deposited in the authors' reference collection (Maassen 2002).

carioca, Oenopota Figueira \& Absalão, 2010

Oenopota carioca Figueira \& Absalão, 2010b: 476 (fig. 1G-I).

Gastropoda, Mangeliidae

Paratypes (3 spc): MZSP 90708.

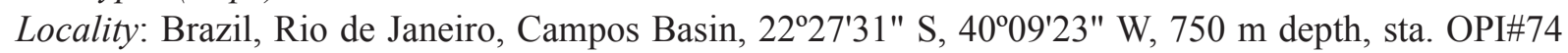
(type locality), 21 Nov. 2002.

Collector: R/V Astro Garoupa.

Preservation: Dry.

cearensis, Cranopsis Simone \& Cunha, 2014

Cranopsis cearensis Simone \& Cunha, 2014: 445-446 (figs 48-55).

Gastropoda, Fissurellidae

Holotype: MZSP 106894.

Paratypes (19 spc): MZSP 90280.

Paratypes (18 spc): MZSP 93940.

Paratypes (2 spc): MZSP 100415.

Locality: Brazil, Ceará, off Fortaleza, Canopus Bank, $2^{\circ} 12^{\prime} 43^{\prime \prime}$ S, $38^{\circ} 18^{\prime} 52^{\prime \prime}$ W, 250 m depth (type locality), Aug. 2005.

Collector: J. Coltro.

Preservation: Dry.

chichiensis, Hemiphaedusa antuensis, Nordsieck, 2005

Hemiphaedusa (Hemizaptyx) antuensis chichiensis Nordsieck, 2005a: 49-50 (pl. 3, fig. 23).

Gastropoda, Clausiliidae

Paratype: MZSP 95497. 
Locality: Taiwan, Hualien Co., W of Chi Chi (ca. $40 \mathrm{~km} \mathrm{~S}$ of Hualien), $23^{\circ} 42.726^{\prime} \mathrm{N}, 121^{\circ} 32.596^{\prime} \mathrm{E}$ (type locality), 13 Apr. 2003.

Preservation: Dry.

Remarks: Former Jens Hemmen Collection. The specimen listed above is a remainder of a 10-specimen paratype lot deposited by Nordsieck in Hemmen's collection.

\section{chodo, Triplodon Mansur \& Pimpão, 2008}

Triplodon chodo Mansur \& Pimpão, 2008: 112-115 (figs 1-6).

Bivalvia, Hyriidae

Paratype: MZSP 86012.

Locality: Brazil, Amazonas, Novo Aripuanã, River Aripuanã, between $06^{\circ} 00^{\prime} 42.3^{\prime \prime} \mathrm{S}, 60^{\circ} 11^{\prime} 42.7^{\prime \prime} \mathrm{W}$ and 0600'21.7" S, 60¹1'45.1" W, 9 Sep. 2004.

Collector: D.M. Pimpão and Sotero.

Preservation: Dry

Remarks: Former INPA 331a.

\section{christae, Opisthostoma Maassen, 2001}

Opisthostoma (Plectostoma) christae Maassen, 2001: 52-53 (figs 3, 10-11).

Gastropoda, Diplommatinidae

Paratype: MZSP 95472 (Fig. 2A-B).

Locality: Malaysia, Kelantan, rd. \#8, Gua Musang to Machang, the right side of km 24.9, 04 $55.022^{\prime} \mathrm{N}$, $102^{\circ} 06.934^{\prime} \mathrm{E}$.

Preservation: Dry.

Remarks: Former Jens Hemmen Collection. Locality and measurements compare fittingly with a 20-specimen paratype lot originally deposited in Hemmen's collection (Maassen 2001).

\section{coltrorum, Conasprelloides Petuch \& Myers, 2014}

Conasprelloides coltrorum Petuch \& Myers, 2014a: 36-37 (fig. 1A-B).

Gastropoda, Conidae

Holotype: MZSP 116141.

Locality: Brazil, Espírito Santo, off Vitória, 60 m depth.

Collectors: Local fishermen.

Preservation: Dry.

Remarks: This species was recombined as Conus coltrorum by Puillandre et al. (2015).

\section{coltrorum, Spixia Simone, 2012}

Spixia coltrorum Simone, 2012b: 433-435 (figs 9-14).

Gastropoda, Orthalicidae

Holotype: MZSP 103920.

Paratype: MZSP 103921.

Paratypes (2 spc): MZSP 103922.

Paratypes (8spc): MZSP 103923.

Locality: Brazil, Bahia, Santa Maria da Vitória, $\sim 13^{\circ} 24^{\prime} \mathrm{S}, 4^{\circ} 12^{\prime} \mathrm{W}, \sim 460 \mathrm{~m}$ altitude (type locality), Jan. 2012.

Collector: J. Coltro.

Preservation: Dry. 


\section{columbaris, Cranopsis Simone \& Cunha, 2014}

Cranopsis columbaris Simone \& Cunha, 2014: 452 (figs 84-89).

Gastropoda, Fissurellidae

Holotype: MZSP 107719.

Paratypes (3 spc): MZSP 105520.

Locality: Brazil, Ceará, off Fortaleza, Canopus Bank, $2^{\circ} 12^{\prime} 43^{\prime \prime}$ S, 38¹8'52" W, 250 m depth (type locality), Aug. 2005.

Collectors: J. Coltro.

Preservation: Dry.

\section{conica, Lacunopsis Brandt, 1968}

Lacunopsis conica Brandt, 1968: 249-250 (pl. 9, fig. 36 + text fig. 27).

Gastropoda, Pomatiopsidae

Probable paratype: MZSP 95495 (Fig. 4E-F).

Locality: Laos, Mekong River, rapids 2 km S of Khone (type locality), 28 Feb. 1968.

Preservation: Dry.

Remarks: Former Jens Hemmen Collection. Data on the specimen's label correspond to the lot originally catalogued as SMRL 16216. The specimen listed above is a possible remainder of a 30-specimen paratype lot left by the author under the care of the SMRL.

\section{conifera, Chrysallida Pimenta, 2012}

Chrysallida conifera Pimenta, 2012: 441-444 (figs 13-28).

Gastropoda, Pyramidellidae

Paratype: MZSP 99920.

Paratype ${ }^{1}$ : MZSP 99921.

Paratype $^{2}$ : MZSP 99923.

Paratype $^{3}$ : MZSP 99924.

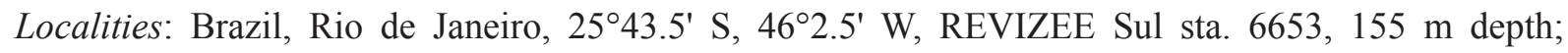
1) $24^{\circ} 00.95^{\prime} \mathrm{S}, 43^{\circ} 55.54^{\prime} \mathrm{W}$, REVIZEE Sul sta. $6662,135 \mathrm{~m}$; 2-3) $24^{\circ} 17.13^{\prime} \mathrm{S}, 44^{\circ} 12.15^{\prime} \mathrm{W}$, REVIZEE Sul sta. 6666, 163 m; all collected between Nov. 1997 and Jan. 1998.

Collector: R/V W. Besnard.

Preservation: Dry.

corallina, Kora Simone, 2012

Kora corallina Simone, 2012b: 432-433 (figs 1-8).

Gastropoda, Orthalicidae

Holotype: MZSP 103910.

Paratype: MZSP 103911.

Paratype: MZSP 103912.

Paratypes (32 spc): MZSP 103913.

Locality: Brazil, Bahia, Santa Maria da Vitória, $\sim 13^{\circ} 24^{\prime}$ S, 441' $\mathrm{W}, \sim 460$ m altitude (type locality), Jan. 2012.

Collector: J. Coltro.

Preservation: Dry. 


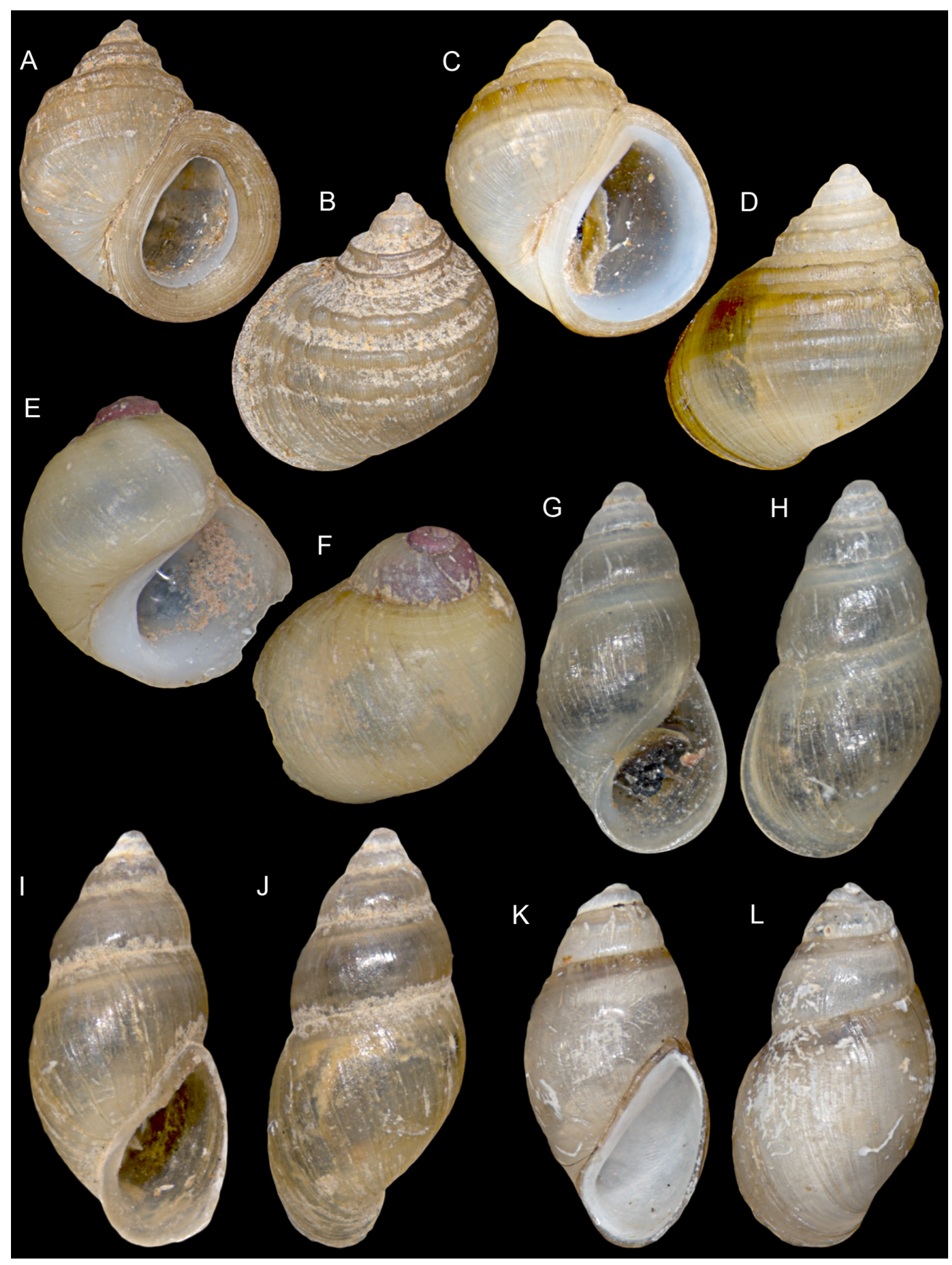

Fig. 4. A-B. Jullienia munensis Brandt, 1974, probable paratopotype MZSP 95482, L = $5.9 \mathrm{~mm}$, $\mathrm{W}=5.2 \mathrm{~mm}$. C-D. Jullienia rolfbrandti Temcharoen, 1971, probable paratype MZSP 95463, $\mathrm{L}=7.3 \mathrm{~mm}, \mathrm{~W}=6 \mathrm{~mm}$. E-F. Lacunopsis conica Brandt, 1968, probable paratype MZSP 95495, $\mathrm{L}=5.3 \mathrm{~mm}, \mathrm{~W}=4.9 \mathrm{~mm}$. G-H. Hubendickia microsculpta Brandt, 1968, probable paratype MZSP 95945, L $=4.35 \mathrm{~mm}, \mathrm{~W}=1.71 \mathrm{~mm}$. I-J. Manningiella polita Brandt, 1970, probable paratype MZSP 95475, L $=4.2 \mathrm{~mm}, \mathrm{~W}=2 \mathrm{~mm}$. K-L. Manningiella rolfbrandti Temcharoen, 1971, probable paratype MZSP 95924, $\mathrm{L}=4.2 \mathrm{~mm}, \mathrm{~W}=2.2 \mathrm{~mm}$. 
corpus, Habeas Simone, 2013

Habeas corpus Simone, 2013b: 520 (figs 1-6, 16-18).

Gastropoda, Diplommatinidae

Holotype: MZSP 110000.

Paratype $^{1}$ : MZSP 106745.

Paratype $^{2}$ : MZSP 106774.

Localities: Brazil, Bahia, Carinhanha, Serra do Ramalho, $400 \mathrm{~m}$ elevation, sta. LES2302, Gruna das

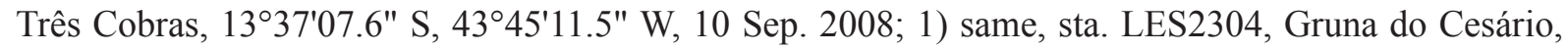
1331'06.1" S, 4338'26.2" W, 12 Sep. 2008; 2) type locality, 10 Sep. 2008.

Collector: M.E. Bichuette.

Preservation: Dry.

\section{crooki, Pachydrobia Brandt, 1968}

Pachydrobia crooki Brandt, 1968: 228-229 (pl. 8, fig. 12 + text fig. 10).

Gastropoda, Pomatiopsidae

Probable paratype: MZSP 95469 (Fig. 2G-H).

Locality: Thailand, Ubon Province, Mekong River at Bandan (type locality), 7 Mar. 1967.

Preservation: Dry.

Remarks: Former Jens Hemmen Collection. Measurements and data on the specimen's label correspond to the lot originally catalogued as SMRL 3431. The specimen listed above is a possible remainder of a 30 -specimen paratype lot left by the author under the care of the SMRL.

\section{crooki, Wykoffia Brandt, 1968}

Wykoffia crooki Brandt, 1968: 245-247 (pl. 9, fig. 33 + text fig. 24).

Gastropoda, Pomatiopsidae

Probable paratype: MZSP 95909.

Locality: East Thailand, Mekong River at Bandan (type locality).

Preservation: Dry.

Remarks: Former Jens Hemmen Collection. Measurements and data on the specimen's label correspond to the lot originally registered under the number SMRL 3463. The specimen listed above is a possible remainder of a 10-specimen paratype lot left by the author under the care of the SMRL. This species was recombined as Jullienia crooki by Davis (1979).

\section{curupira, Yoldiella Benaim \& Absalão, 2011}

Yoldiella curupira Benaim \& Absalão, 2011: 523-525 (fig. 8A-H).

Bivalvia, Yoldiidae

Paratypes (2 valves): MZSP 93359.

Locality: Brazil, Rio de Janeiro, Campos Basin, sta. 41, 22³9'34" S, 4008'22" W, 1200 m depth.

Collector: R/V Astro-Garoupa.

Preservation: Dry.

cylindrata, Eulimella Pimenta, Santos \& Absalão, 2011

Eulimella cylindrata Pimenta, Santos \& Absalão, 2011: 30 (figs 26-31).

Gastropoda, Pyramidellidae

Paratypes (2 spc): MZSP 101568. 
Locality: Brazil, off Espírito Santo, 2132' S, 4009' W, sta. CB96, 295-300m depth (type locality), 31 May 1987.

Collectors: P. Bouchet, J.H. Leal and B. Métivier.

Preservation: Dry.

Remarks: Former MNHN, Paris.

cylindrica, Hubendickia Brandt, 1974

Hubendickia cylindrica Brandt, 1974: 96-97 (pl. 7, fig. 19).

Gastropoda, Pomatiopsidae

Probable paratype: MZSP 95944 (Fig. 3C-D).

Locality: Thailand, Ubon Province, Mekong River at Bandan (type locality), 15 Feb. 1970.

Preservation: Dry.

Remarks: Former Jens Hemmen Collection. Given its compatible measurements, the specimen listed above is a possible remainder of a 100 -specimen paratype lot from the above-mentioned locality, originally catalogued as SMRL 5099. According to Brandt (1974), these specimens were left under the care of the SMRL.

damasomonteiroi, Jaspidiconus Petuch \& Myers, 2014

Jaspidiconus damasomonteiroi Petuch \& Myers, 2014b: 35 (text fig. + fig. 3A-F).

Gastropoda, Conidae

Holotype: MZSP 116528.

Paratype: MZSP 116529.

Locality: Brazil, Ceará State, off Camocim, $40 \mathrm{~m}$ depth.

Preservation: Dry.

Remarks: This species was recombined as Conasprella damasomonteiroi by Puillandre et al. (2015).

\section{danieli, Astraea Alf \& Kreipl, 2006}

Astraea (Astralium) danieli Alf \& Kreipl, 2006: 91 (figs 1-3).

Gastropoda, Turbinidae

Paratype: MZSP 95919 (Fig. 1A-B).

Locality: Indonesia, Bali, Tanah Lot. In shallow water on rocks with algae, collected in 1978.

Collectors: A. Alf and K. Krepl.

Preservation: Dry.

Remarks: One of the five paratypes deposited in Jens Hemmen's Collection by Alf \& Kreipl. This species was recombined as Astralium danieli by Alf \& Kreipl (2011).

data, Habeas Simone, 2013

Habeas data Simone, 2013b: 520-522 (figs 7-10, 16, 18).

Gastropoda, Diplommatinidae

Holotype: MZSP 106810.

Paratypes $^{1}$ (2 spc): MZSP 106814.

Paratype $^{2}$ : MZSP 109965.

Paratype $^{3}$ : MZSP 110159.

Localities: Brazil, Bahia, Carinhanha, Serra do Ramalho, $400 \mathrm{~m}$ elevation, sta. LES2301, Gruna do

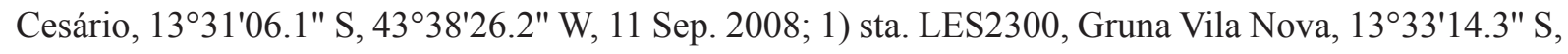
4352'40.7" W; 2) sta. LES2304, 12 Sep. 2008; 3) 12 Sep. 2008.

Collector: M.E. Bichuette.

Preservation: Dry. 


\section{decresca, Austromitra Simone \& Cunha, 2012}

Austromitra decresca Simone \& Cunha, 2012: 765-766 (fig. 7A-D).

Gastropoda, Costellariidae

Paratype: MZSP 102687.

Locality: Brazil, Espírito Santo, off Itaúnas, Abrolhos Slope, $18^{\circ} 56^{\prime}$ S, $37^{\circ} 52^{\prime}$ W, MD55 sta. DC82, 85-105 m depth, 28 May 1987.

Collectors: P. Bouchet, J.H. Leal and B. Métivier.

Preservation: Dry.

denudata, Profundisepta Simone \& Cunha, 2014

Profundisepta denudata Simone \& Cunha, 2014: 457-458 (figs 109-115).

Gastropoda, Fissurellidae

Holotype: MZSP 105507.

Paratype: MZSP 105508.

Locality: Brazil, São Paulo, continental slope off Ubatuba, $24^{\circ} 12^{\prime} \mathrm{S}, 40^{\circ} 23^{\prime} \mathrm{W}, 3049 \mathrm{~m}$ depth, R/V W. Besnard sta. PI (type locality), 1 Jun. 1971.

Collectors: Researchers onboard R/V W. Besnard.

Preservation: Dry.

diabula, Oenopota Figueira \& Absalão, 2010

Oenopota diabula Figueira \& Absalão, 2010b: 475-476 (fig. 1D-F).

Gastropoda, Mangeliidae

Paratype': MZSP 90706.

Paratypes $^{2}$ (2 spc): MZSP 90707.

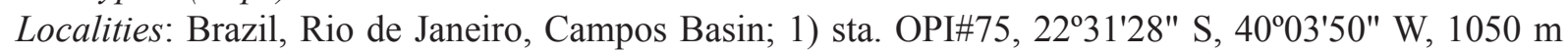
depth, 19 Nov. 2002; 2) sta. OPII\#84, 22²6'27" S, 3958'51" W, 1050 m, 20 Jun. 2003.

Collector: R/V Astro Garoupa.

Preservation: Dry.

\section{domaneschii, Cyamiocardium Passos \& Machado, 2014}

Cyamiocardium domaneschii Passos \& Machado, 2014: 123-126 (figs 1-5).

Bivalvia, Cyamiidae

Paratypes $^{1}$ (5 spc): MZSP 114207.

Paratypes $^{2}$ (8 spc): MZSP 114208.

Paratypes $^{3}$ (7 spc): MZSP 114209.

Localities: Brazil, off Rio de Janeiro and Espírito Santo; 1-2) sta. HAB11 A-01 R3, 22 55'7.555" S, $42^{\circ} 0^{\prime} 49.218^{\prime \prime} \mathrm{W}$; 3) sta. HAB11 D-02 R2, 22॰12'53.045" S, 4051'12.031" W.

Collectors: HABITATS Project researchers.

Preservation: Dry.

\section{ejuncida, Eulimella Pimenta, Santos \& Absalão, 2011}

Eulimella ejuncida Pimenta, Santos \& Absalão, 2011: 32-33 (figs 40-47).

Gastropoda, Pyramidellidae

Holotype: MZSP 99916.

Paratypes (4 spc): MZSP 99917.

Paratypes $^{1}$ (5 spc): MZSP 99918.

Paratypes $^{2}$ (3 spc): MZSP 99919. 


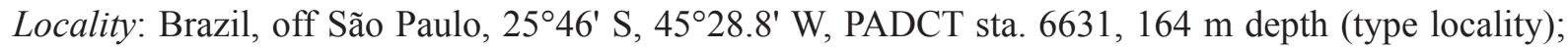
1) off Paraná, $24^{\circ} 00.95^{\prime} \mathrm{S}, 43^{\circ} 55.54^{\prime} \mathrm{W}$, REVIZEE sta. 6662, $135 \mathrm{~m}$; 2) off Rio de Janeiro, $23^{\circ} 49.07^{\prime} \mathrm{S}$, $42^{\circ} 46.81^{\prime} \mathrm{W}$, PADCT sta. $6553,225 \mathrm{~m}$.

Preservation: Dry.

\section{elegantissima, Symptychiella Nordsieck, 2005}

Symptychiella (Divanenia) elegantissima Nordsieck, 2005b: 213-214 (fig. 22).

Gastropoda, Clausiliidae

Paratype: MZSP 95488.

Locality: Peru, Dept. San Martin, path from Palestina to Cueva Huacharos de Palestina (ca. 23 km NW

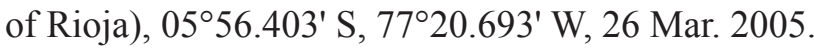

Collector: J. Hemmen.

Preservation: Dry.

Remarks: Former Jens Hemmen Collection. The specimen listed above is a remainder of a 13 -specimen paratype lot deposited by Nordsieck in Hemmen's collection.

elisabethae, Codringtonia (Codringtonia) Subai, 2005

Codringtonia (Codringtonia) elisabethae Subai, 2005: 71-74 (pl. 5, fig. $29+$ text figs 2, 10, 11).

Gastropoda, Helicidae

Probable paratype: MZSP 95950.

Locality: Greece, NE Peloponnese, Argos, castle, 28 Mar. 1985.

Collector: J. Hemmen.

Preservation: Dry.

Remarks: Former Jens Hemmen Collection. As per measurements and label data, the specimen listed above is a possible remainder a three-specimen paratype lot collected by Hemmen. According to Subai (2005: 74), these specimens should have been sent to W.J.M. Maassen's private collection.

\section{elisae, Lucapina Costa \& Simone, 2006}

Lucapina elisae Costa \& Simone, 2006: 1-4 (figs 1-18).

Gastropoda, Fissurellidae

Paratypes (3 spc): MZSP 53934.

Paratypes (9 spc): MZSP 61229.

Paratypes (3 spc): MZSP 67299.

Paratype: MZSP 78194.

Locality: Brazil, Ceará, off Fortaleza, Canopus Bank, 02¹4'25" S, 38²2'50" W, 240-260 m depth, Nov. 2005.

Collectors: J. Coltro and P.M. Costa.

Preservation: Dry; MZSP 61229 is preserved in 70\% ethanol (soft parts).

enceladus, Benthomangelia Figueira \& Absalão, 2010

Benthomangelia enceladus Figueira \& Absalão, 2010a: 735-736 (fig. 1D-F).

Gastropoda, Mangeliidae

Paratype: MZSP 90703.

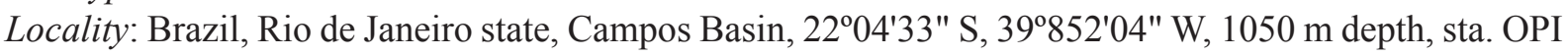
\#50, 24 Nov. 2002.

Collector: R/V Astro Garoupa.

Preservation: Dry. 


\section{enigmatica, Cranopsis Simone \& Cunha, 2014}

Cranopsis enigmatica Simone \& Cunha, 2014: 444-445 (figs 42-47).

Gastropoda, Fissurellidae

Paratype: MZSP 102941.

Locality: Brazil, Espírito Santo, off Regência, Abrolhos Slope, 1940' S, 37²48' W, 790-940 m, MD55 sta. CB77 (type locality), 27 May 1987.

Collectors: P. Bouchet, J.H. Leal and B. Métivier.

Preservation: Dry.

Remarks: Former MNHN, Paris.

equatorialis, Thylaeodus Spotorno \& Simone, 2013

Thylaeodus equatorialis Spotorno \& Simone, 2013: 89-91 (figs 1-18).

Gastropoda, Vermetidae

Paratypes $^{1}$ (5 spc): MZSP 82314.

Paratypes $^{2}$ (3 spc): MZSP 86531.

Paratypes $^{3}$ (85 spc): MZSP 86566.

Paratypes $^{4}$ (32 spc): MZSP 86627.

Paratypes $^{5}$ (32 spc): MZSP 86628.

Paratypes $^{6}$ (2 spc): MZSP 86676.

Paratypes $^{7}$ (3 spc): MZSP 87469.

Locality: Brazil, Pernambuco, São Pedro e São Paulo Archipelago, Belmonte Inlet, Enseada Beach, $00^{\circ} 55^{\prime} 01^{\prime \prime} \mathrm{N}, 29^{\circ} 20^{\prime} 44^{\prime \prime} \mathrm{W}, 3-10 \mathrm{~m}$ depth (type locality); 1) Mar. 2005; 2) 28 May 2007; 3$) 7$ Jun. 2007;

4) 7 Nov. 2007; 5) 7 Nov. 2007; 6) 9 Nov. 2007; 7) 27 Oct. 2007.

Collectors: L.R. Simone and C.M. Cunha.

Preservation: All 70\% ethanol; single specimen (MZSP 86566) is preserved dry.

\section{ericmonnieri, Jaspidiconus Petuch \& Myers, 2014}

Jaspidiconus ericmonnieri Petuch \& Myers, 2014a: 42 (fig. 2G-I).

Gastropoda, Conidae

Holotype: MZSP 116144.

Locality: Brazil, Espírito Santo State, off Vitória, $60 \mathrm{~m}$ depth.

Collectors: Local fishermen.

Preservation: Dry.

Remarks: This species was recombined as Conasprella ericmonnieri by Puillandre et al. (2015).

exiguum, Eulimastoma Pimenta, 2012

Eulimastoma exiguum Pimenta, 2012: 444 (figs 19-24).

Gastropoda, Pyramidellidae

Paratypes (4 spc): MZSP 105121.

Locality: Brazil, Rio de Janeiro, East of Cape São Tomé, 21³5' S, 40³1' W, MD55 sta. CB98, 900 m depth, 31 May 1987.

Collector: P. Bouchet, J.H. Leal and B. Métivier.

Preservation: Dry.

Remarks: Former MNHN, Paris. 


\section{fetidus, Leiostracus Salvador \& Cavallari, 2014}

Leiostracus fetidus Salvador \& Cavallari, 2014: 40 (figs 2-8).

Gastropoda, Orthalicidae

Holotype: MZSP 112123.

Paratypes (13 spc): MZSP 107577.

Locality: Brazil, Bahia, Atlantic Rainforest fragment near the city of Canavieiras, $15^{\circ} 40^{\prime} \mathrm{S}, 39^{\circ} 02^{\prime} \mathrm{W}$ (type locality), Jun. 2012.

Collectors: A. Bianchi.

Preservation: Dry.

\section{fischerpiettei, Paraprososthenia Brandt, 1970}

Paraprososthenia fischerpiettei Brandt, 1970: 187-188 (pl. 13, fig. $5+$ text fig. 5).

Gastropoda, Pomatiopsidae

Probable paratype: MZSP 95496 (Fig. 2K-J).

Locality: Laos, Sedone River at Khong Sedone, 23 Feb. 1968.

Collector: P. Temcharoen.

Preservation: Dry.

Remarks: Former Jens Hemmen Collection. Measurements and data on the specimen's label correspond to the lot originally catalogued as SMRL 16118. The specimen listed above is a possible remainder of a 50-specimen paratype lot from the above-mentioned locality. According to Brandt (1970), these specimens were left under the care of the SMRL.

\section{gaiophanis, Microlinices Simone, 2014}

Microlinices gaiophanis Simone, 2014: 580-582 (fig. 8H-K).

Gastropoda, Naticidae

Paratype: MZSP 105127.

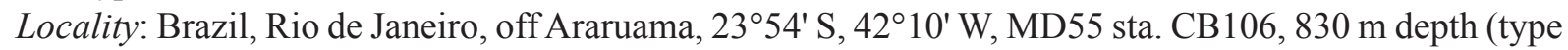
locality), 2 Jun. 1987.

Collectors: P. Bouchet, J.H. Leal and B. Métivier.

Preservation: Dry.

Remarks: Former MNHN, Paris.

gaucha, Eledone Haimovici, 1988

Eledone gaucha Haimovici, 1988: 82-84 (figs 2-14).

Cephalopoda, Octopodidae

Paratypes (2 spc): MZSP 25242 (Fig. 7E).

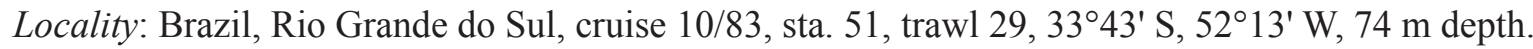

Collector: R/V Atlâtico Sul, M. Haimovici.

Preservation: $70 \%$ ethanol.

\section{gibbosula, Temesa Nordsieck, 2005}

Temesa gibbosula Nordsieck, 2005b: 208-209 (fig. 10).

Gastropoda, Clausiliidae

Paratype: MZSP 95926.

Locality: Peru, Junin Department, c. $2 \mathrm{~km}$ from Tarma to La Oroya, c. $4000 \mathrm{~m}$ altitude, $11^{\circ} 24.283^{\prime} \mathrm{S}$, $75^{\circ} 49.048^{\prime} \mathrm{W}$ (type locality), 15 Oct. 2004. 
Collector: J. Hemmen.

Preservation: Dry.

Remarks: Former Jens Hemmen Collection. The specimen listed above is a remainder of a 9-specimen paratype lot deposited by Nordsieck in Hemmen's collection. This species was recombined as Parabalea gibbosula by Nordsieck (2010).

\section{gilbertus, Rhinus Simone \& Casati, 2013}

Rhinus gilbertus Simone \& Casati, 2013: 153-154 (figs 23-34).

Gastropoda, Simpulopsidae

Holotype: MZSP 112449.

Paratypes $^{1}$ (27 spc): MZSP 111827.

Paratypes $^{2}$ (50 spc): MZSP 111839.

Paratypes $^{3}$ (9 spc): MZSP 111844.

Localities: Brazil, Piauí, Serra da Capivara, Coronel José Dias Municipality, close to Sítio do Mocó Town, Toca de Cima dos Pilão Cave, 851'47.10" S, 42³3'26.96" W; 1-2) same, sta. P2; 3) same, sta. P1.

Collector: R. Casati.

Preservation: Dry.

Remarks: Specimens in the lot MZSP 111835 are listed as paratypes of Rhinus gilbertus by Simone \& Casati (2013, original description), but are in fact topotypes of C. capivara, which is described in the same paper. Simone \& Casati (2013) simultaneously reported the catalogue number MZSP 111845 among the paratypes of Rhinus gilbertus and C. capivara. This catalogue number does not contain paratypes of $R$. gilbertus; this information is incorrect in the original description and should be disregarded.

\section{gili, Pazinotus Costa \& Pimenta, 2012}

Pazinotus gili Costa \& Pimenta, 2012: 121-123 (figs 5-6).

Gastropoda, Muricidae

Paratypes (2 spc): MZSP 70282.

Paratypes (3 spc): MZSP 70335.

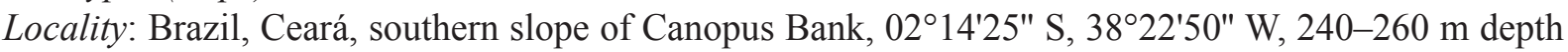
(type locality), Oct.-Nov. 2005.

Collector: J. Coltro.

Preservation: Dry.

\section{gochenouri, Hubendickia Brandt, 1968}

Hubendickia gochenouri Brandt, 1968: 238 (pl. 8, fig. 25).

Gastropoda, Pomatiopsidae

Probable paratype: MZSP 95943.

Locality: Thailand, Mekong at Ban Kum (type locality), 5 Sep. 1967.

Preservation: Dry.

Remarks: Former Jens Hemmen Collection. Data on the specimen's label correspond to the lot originally catalogued as SMRL 3385. The specimen listed above is a possible remainder of a 10-specimen paratype lot left by the author under the care of the SMRL.

goudi, Teracharopa Maassen, 2000

Teracharopa goudi Maassen, 2000: 142-144 (figs 9-10).

Gastropoda, Charopidae 
Probable paratype: MZSP 95494 (Fig. 7A-B).

Locality: Indonesia, West Sumatra, SE of Bukit Tingii, 3 km E of Lintau, Cave Gua Pangian, 00²8'19.5" S, $100^{\circ} 45^{\prime} 11.7^{\prime \prime}$ E (type locality).

Preservation: Dry.

Remarks: Former Jens Hemmen Collection. Locality data and measurements compare fittingly with a 20-specimen paratype series originally deposited in the authors' reference collection (Maassen 2000). Label bears a paratype status annotation.

\section{hansrosaorum, Hoplodoris Domínguez, García \& Troncoso, 2006}

Hoplodoris hansrosaorum Domínguez, García \& Troncoso, 2006: 150-152 (figs 1-14).

Gastropoda, Discodorididae

Holotype: MZSP 52190.

Locality: Brazil, Rio de Janeiro, Arraial do Cabo, Cabo Frio Island, interdital zone (type locality), 28 Jul. 1999.

Collectors: J. Troncoso and García.

Preservation: $70 \%$ ethanol.

\section{hazinorum, Conasprelloides Petuch \& Myers, 2014}

Conasprelloides hazinorum Petuch \& Myers, 2014b: 31 (text fig. + fig. 1A-F).

Gastropoda, Conidae

Holotype: MZSP 116523.

Paratype: MZSP 116524.

Locality: Brazil, Sergipe State, off Pirambu, $50 \mathrm{~m}$ depth.

Preservation: Dry.

Remarks: This species was recombined as Conus hazinorum by Puillandre et al. (2015).

\section{helenae, Anachis Costa, 1983}

Anachis helenae Costa, 1983: 95-98 (figs 1-3).

Gastropoda, Columbellidae

Holotype: MZSP 102531 (Fig. 5E-F).

Paratype: MZSP 102534.

Paratype 2 : MZSP 102539.

Localities: Brazil, Rio de Janeiro, Niterói off Boa Viagem Island, 22 $2^{\circ} 54^{\prime} 43^{\prime \prime} \mathrm{S}, 43^{\circ} 07^{\prime} 32^{\prime \prime}$ W, 8 m depth, Aug. 1979; 1-2) Brazil off Amapá State, 56 m, 1 May 1968.

Collectors: P. M. Costa; 1-2) R/V Almirante Saldanha.

Preservation: Dry. All specimens including the holotype suffered the effects of Bynesian decay due to poor storage conditions prior to donation. Specimens were reconditioned according to the recommendations of Cavallari et al. (2014).

Remarks: Former USU. The holotype was formerly catalogued as USU 215; paratype ${ }^{1}$ as USU 237; paratype $^{2}$ as USU 238. The specimens listed above were donated to the MZSP in 2011.

\section{henriquei, Coltroconus Petuch \& Myers, 2014}

Coltroconus henriquei Petuch \& Myers, 2014a: 40 (fig. 2A-C).

Gastropoda, Conidae

Holotype: MZSP 116143.

Locality: Brazil, Bahia State, east of the Abrolhos Platform, western side of Royal Charlotte Bank, 35 m depth. 

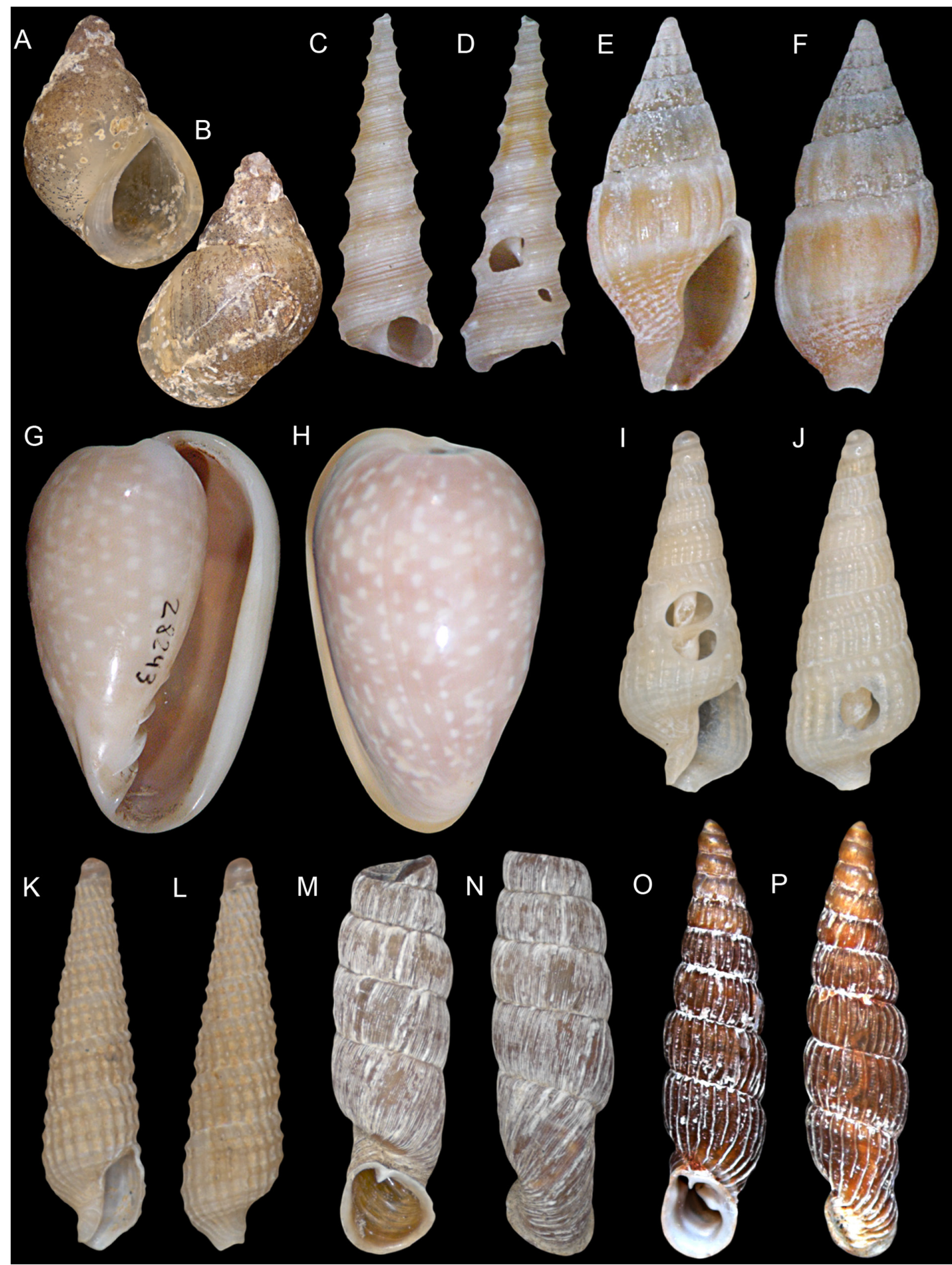

Fig. 5. A-B. Lithoglyphopsis aperta Temcharoen, 1971, Probable paratype MZSP 95942, L $=2.75 \mathrm{~mm}$, $\mathrm{W}=2 \mathrm{~mm}$. C-D. Turritella ahiparana Powell, 1927, Probable paratype MZSP 101757, L $=12.7 \mathrm{~mm}$, $\mathrm{W}=4.2 \mathrm{~mm}$. E-F. Anachis helenae Costa, 1983, holotype MZSP 102531, L = $10.3 \mathrm{~mm}, \mathrm{~W}=4.5 \mathrm{~mm}$. G-H. Bullata analuciae Souza \& Coovert, 2001, paratype MZSP 28243, L = $31.8 \mathrm{~mm}, \mathrm{~W}=19.7 \mathrm{~mm}$. I-J. Terebra intumescyra Lima, Tenório \& Barros, 2007, paratype MZSP 84237, L =5.4 mm, W= $2 \mathrm{~mm}$. K-L. Terebra simonei Lima, Tenório \& Barros, 2007, paratype MZSP 84235, L = 5.4 mm, W = $1.4 \mathrm{~mm}$. M-N. Andiniella flammulata unicolor Nordsieck, 2005, paratype MZSP 95490, L =12.5 mm, W=3.8 mm. O-P. Boettgeria jensi Neubert \& Groh, 1998, paratype MZSP 95947, L = $11.3 \mathrm{~mm}, \mathrm{~W}=2.8 \mathrm{~mm}$. 
Preservation: Dry.

Remarks: This species was recombined as Conasprella henriquei by Puillandre et al. (2015).

\section{hemitoma, Hemimarginula Simone \& Cunha, 2014}

Hemimarginula hemitoma Simone \& Cunha, 2014: 463-464 (figs 140-151).

Gastropoda, Fissurellidae

Paratype: MZSP 101247.

Paratypes $^{1}$ (2 spc): MZSP 102947.

Paratypes $^{2}$ (3 spc): MZSP 102948.

Locality: Brazil, off Espírito Santo, Trindade Island, Andradas Beach, 20³0'50" S, 29¹8'20" W, 0-1 m depth; 1) Espírito Santo, off Itaúnas, Abrolhos Slope, 1850' S, 3757' W, 60 m, MD55 sta. DC83 (type locality), 28 May 1987; 2) 1856' S, 3752' W, 85-105 m, MD55 sta. DC82, 28 May 1987.

Collectors: P. Bouchet, J.H. Leal \& B. Métivier.

Preservation: Dry.

Remarks: Former MNHN, Paris.

\section{hemmeni, Cyclonenia Nordsieck, 2005}

Cyclonenia hemmeni Nordsieck, 2005b: 206 (fig. 5).

Gastropoda, Clausiliidae

Paratype: MZSP 95477 (Fig. 6A-B).

Locality: Peru, Cajamar Department, c. Km 148.2 rd. Pacasmayo-Cajamarca, 2500 m altitude (type locality), 27 Oct. 2004.

Collector: J. Hemmen.

Preservation: Dry.

Remarks: Former Jens Hemmen Collection. The specimen listed above is a remainder of a 28 -specimen paratype lot deposited by Nordsieck in Hemmen's collection.

\section{herndli, Jaspidiconus Petuch \& Myers, 2014}

Jaspidiconus herndli Petuch \& Myers, 2014a: 42-43 (fig. 3A-C).

Gastropoda, Conidae

Holotype: MZSP 116151.

Locality: The Bahamas, western Great Bahama Bank, Bimini Chain, $3 \mathrm{~km}$ due east of Victory Cay, $5 \mathrm{~m}$ depth.

Preservation: Dry.

Remarks: This species was recombined as Conasprella herndli by Puillandre et al. (2015).

\section{honkerorum, Gradiconus Petuch \& Myers, 2014}

Gradiconus honkerorum Petuch \& Myers, 2014a: 37 (fig. 1C-D).

Gastropoda, Conidae

Holotype: MZSP 116148.

Locality: Bahamas, Bahama Bank, Abaco Islands, Green Turtle Cay, 2 m depth.

Collector: P. Honker.

Preservation: Dry.

Remarks: This species was recombined as Conus honkerorum by Puillandre et al. (2015). 

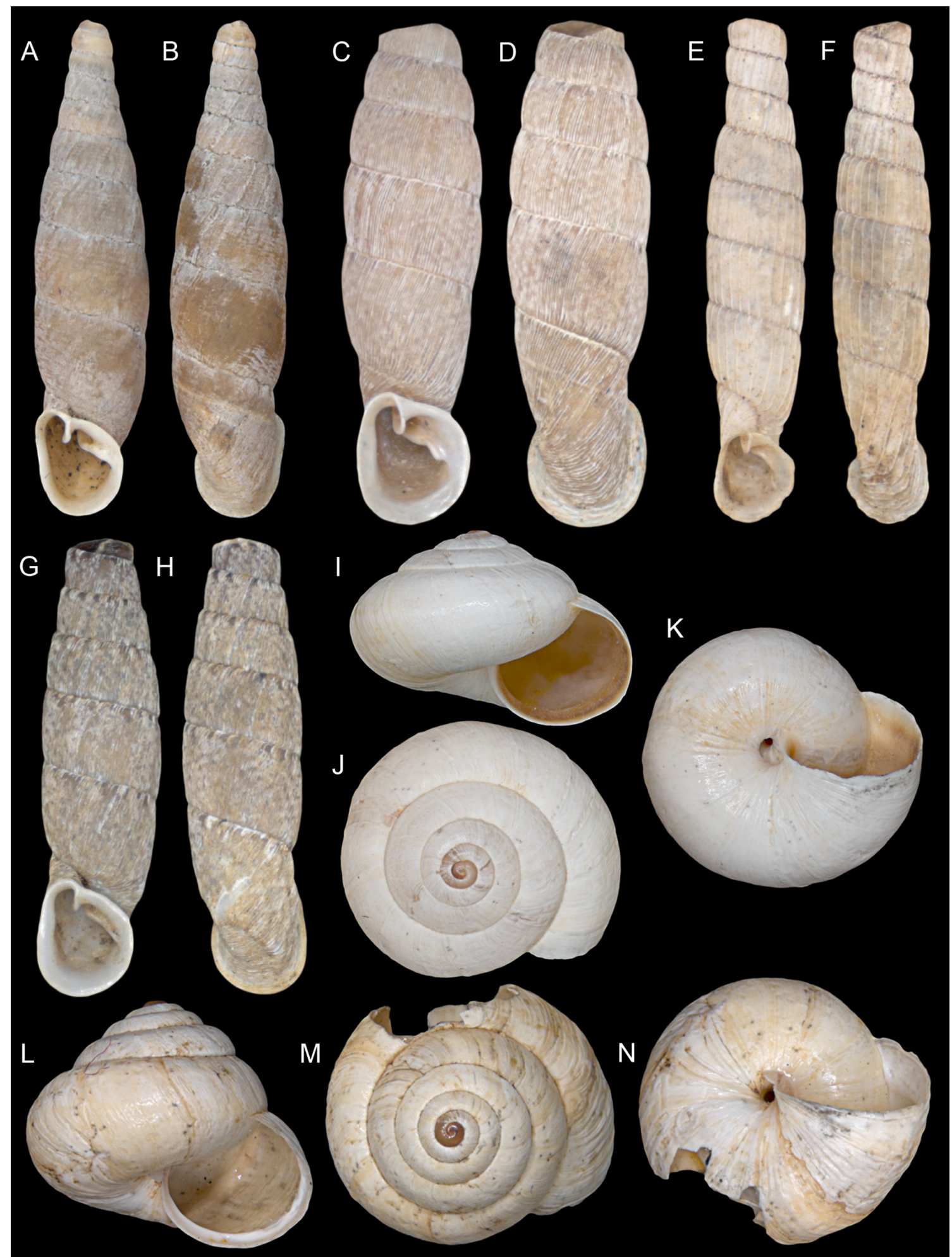

Fig. 6. A-B. Cyclonenia hemmeni Nordsieck, 2005, paratype MZSP 95477, L $=18 \mathrm{~mm}, \mathrm{~W}=4 \mathrm{~mm}$. C-D. Cylindronenia pangamitoensis pongoensis Nordsieck, 2005, paratype MZSP 95470, L = $13.2 \mathrm{~mm}$, $\mathrm{W}=3.6 \mathrm{~mm}$. E-F. Neniella strictecostata Nordsieck, 2005, paratype MZSP 95947, L $=16.8 \mathrm{~mm}$, $\mathrm{W}=3.3 \mathrm{~mm}$. G-H. Steeriana nivea Nordsieck, 2005, paratype MZSP 95479, L = $16.9 \mathrm{~mm}, \mathrm{~W}=4.6 \mathrm{~mm}$. I-K. Cernuella selmaniana Brandt, 1959, probable paratype MZSP 95484, L = 11.6 mm, W = $16.4 \mathrm{~mm}$. L-N. Trochoidea regimaensis Brandt, 1959, probable paratype MZSP 95910, L = 10.2 mm, W $=12 \mathrm{~mm}$. 


\section{hospitalis, Hydrorissoia Brandt, 1968}

Hydrorissoia hospitalis Brandt, 1968: 244-245 (pl. 9, fig. $32+$ text fig. 23).

Gastropoda, Pomatiopsidae

Probable paratype: MZSP 95949 (Fig. 3I-J).

Locality: Thailand, Khong Island, Mekong at Khong Village (type locality), 22 Feb. 1968.

Preservation: Dry.

Remarks: Former Jens Hemmen Collection. Data on the specimen's label correspond to the lot originally catalogued as SMRL 3479. The specimen listed above is a possible remainder of 3 paratypes left by the author under the care of the SMRL. This species is a synonym of Hydrorissoia gracilis Brandt, 1968 according to Davis (1979).

\section{ibitingus, Microlinices Simone, 2014}

Microlinices ibitingus Simone, 2014: 573-574 (figs 4A-G, 5A-F, 11F-G).

Gastropoda, Naticidae

Paratypes (3 spc): MZSP 105250.

Paratypes $^{1}$ (3 spc): MZSP 105251.

Paratypes $^{2}$ (6 spc): MZSP 105252.

Localities: Brazil, Espírito Santo, off Regência, 1940' S, 37048' W, MD55 sta. CB77, 790-940 m depth (type locality), 27 May 1987; 1) off Itaúnas, Abrolhos Slope, 1859' S, 3748' W, MD55 sta. DC73, 607-620 m, 27 May 1987; 2) off Conceição da Barra, Abrolhos Slope, 1858' S, 3749' W, MD55 sta. CB76, 637 m, 27 May 1987.

Collectors: P. Bouchet, J.H. Leal and B. Métivier.

Preservation: Dry.

Remarks: Former MNHN, Paris.

\section{icosisculpta, Emarginula Simone \& Cunha, 2014}

Emarginula icosisculpta Simone \& Cunha, 2014: 455-456 (figs 135-139).

Gastropoda, Fissurellidae

Paratype: MZSP 102949.

Localities: Brazil, Espírito Santo, Trindade Island, Enseada dos Portugueses, $20^{\circ} 30^{\prime} 15^{\prime \prime} \mathrm{S}, 29^{\circ} 18^{\prime} 46^{\prime \prime} \mathrm{W}$, 10-12 m depth, MD55 sta. 1 (type locality), May 1987.

Collectors: P. Bouchet, J.H. Leal and B. Métivier.

Preservation: Dry.

Remarks: Former MNHN, Paris.

\section{insolita, Clinispira Simone \& Casati, 2013}

Clinispira insolita Simone \& Casati, 2013: 147-149 (figs 2-15).

Gastropoda, Odontostomidae

Holotype: MZSP 111847.

Paratypes $^{1}$ (84 spc): MZSP 111828.

Paratypes $^{2}$ (5 spc): MZSP 111829.

Paratypes $^{3}$ (5 spc): MZSP 111830.

Paratypes $^{4}$ (4 spc): MZSP 111831.

Paratypes $^{5}$ (5 spc): MZSP 111832.

Paratype $^{6}$ : MZSP 111833.

Paratypes $^{7}$ (2 spc): MZSP 111834.

Paratypes $^{8}$ (8 spc): MZSP 111846. 
Paratypes $^{9}$ (3 spc): MZSP 112369.

Paratype $^{10}$ : MZSP 112426.

Localities: Brazil, Piauí, Serra da Capivara, Coronel José Dias Municipality, close to Sítio do Mocó Town, Toca de Cima dos Pilão Cave, 8$\left.{ }^{\circ} 51^{\prime} 47.10^{\prime \prime} \mathrm{S}, 4^{\circ} 33^{\prime} 26.96^{\prime \prime} \mathrm{W} ; 1\right)$ same, sta. P4; 2-3) same, sta. P3; 4) same, sta. P2; 5-8) same, sta. P1; all collected in 2012; 9-10) from type locality.

Collector: R. Casati.

Preservation: Dry.

intumescyra, Terebra Lima, Tenório \& Barros, 2007

Terebra intumescyra Lima, Tenório \& Barros, 2007: 65-66 (figs 8-12).

Gastropoda, Terebridae

Holotype: MZSP 84236.

Paratype: MZSP 84237 (Fig. 5I-J).

Locality: Brazil, off Rio Grande do Norte, $04^{\circ} 51^{\prime} 40^{\prime \prime}$ S, 3506'01" W, D-23, 384 m depth (type locality), 24 Nov. 2001.

Collector: R/V Natureza.

Preservation: Dry.

irakita, Euglandina Jardim, Abbate \& Simone, 2013

Euglandina irakita Jardim, Abbate \& Simone, 2013: 327-329 (figs 1-5).

Gastropoda, Spiraxidae

Holotype: MZSP 97238.

Locality: Brazil, Pará, Tapirapé-Aquiri National Forest, 0256'00" S, 51ํ5'00" W, 15 Sep. 2010.

Collectors: J. Jardim and D. Abbate.

Preservation: Dry.

itanhura, Episcynia Simone, 2012

Episcynia itanhura Simone, 2012c: 81-83 (figs 16-27).

Gastropoda, Tornidae

Holotype: MZSP 106553.

Paratypes (18 spc): MZSP 106556.

Locality: Brazil, Espírito Santo, São Mateus, off Guriri, $18^{\circ} 47^{\prime} \mathrm{S}, 32^{\circ} 39^{\prime} \mathrm{W}, 3$ m depth (type locality).

Preservation: Dry, 3 paratypes with metallic coating due to SEM examination.

jensi, Arinia Maassen, 2001

Arinia jensi Maassen, 2001: 56 (figs 5, 14-15).

Gastropoda, Diplommatinidae

Paratype: MZSP 95939.

Locality: Peninsular Malaysia, Kelantan, along the right side of road 8 from Gua Musang to Machamg at km 24.9; 0455'022" N, 10206'934" E.

Collectors: J. and C. Hemmen.

Preservation: Dry.

Remarks: Former Jens Hemmen Collection. The specimen listed above is a remainder of a 250 -specimen paratype lot deposited by the author in Hemmen's collection (Maassen 2001). 


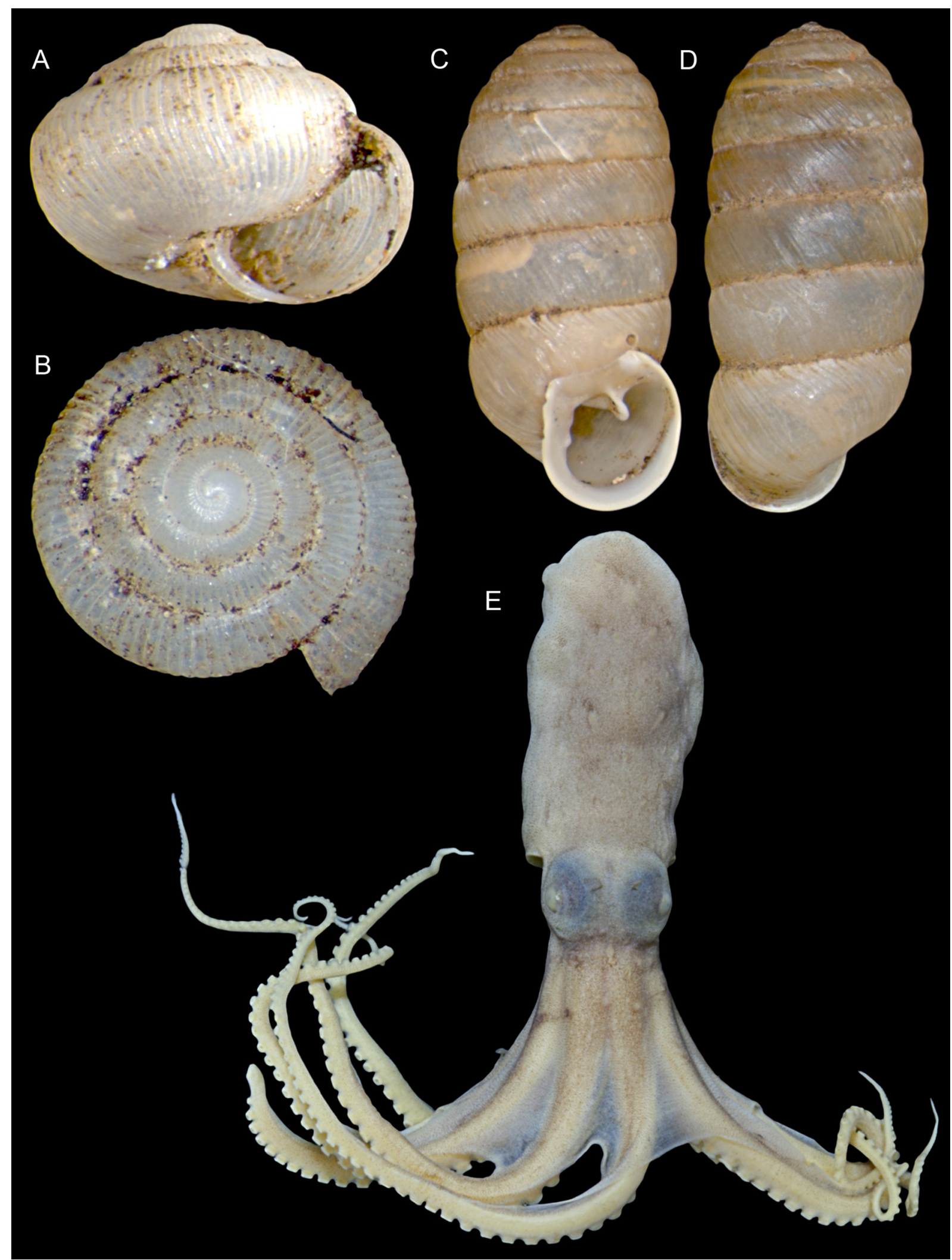

Fig. 7. A-B. Teracharopa goudi Maassen, 2000, probable paratype MZSP 95494, L =2 mm, W = $2.7 \mathrm{~mm}$. C-D. Orculella astirakiensis Gittenberger \& Hausdorf, 2004, paratype MZSP 95489, L = 8.3 mm, W= $4 \mathrm{~mm}$. E. Eledone gauch Haimovici, 1988, paratype MZSP 25242, approximate mantle length $=30 \mathrm{~mm}$. 


\section{jensi, Boettgeria Neubert \& Groh, 1998}

Boettgeria (Boettgeria) jensi Neubert \& Groh, 1998: 159-162 (fig. 3).

Gastropoda, Clausiliidae

Paratype: MZSP 95947 (Fig. 5O-P).

Locality: Portugal, Madeira Archipelago, Desertas Island, 29-31 Jul. 1985.

Collectors: J. Gerber, K. Groh and J. Hemmen.

Preservation: Dry.

Remarks: Former Jens Hemmen Collection. The specimen listed above is a remainder of a 17 -specimen paratype lot deposited by Neubert \& Groh in Hemmen's collection.

\section{joapyra, Subcancilla Simone \& Cunha, 2012}

Subcancilla joapyra Simone \& Cunha, 2012: 766-768 (fig. 7A-D).

Gastropoda, Mitridae

Paratype: MZSP 102675.

Locality: Brazil, Espírito Santo, off Itaúnas, Abrolhos Slope, 1859' S, 3750' W, MD55 sta. DC75, 295 m depth, 27 May 1987.

Collectors: P. Bouchet, J.H. Leal and B. Métivier.

Preservation: Dry.

Remarks: Former MNHN, Paris.

\section{jukyry, Turricostellaria Simone \& Cunha, 2012}

Turricostellaria jukyry Simone \& Cunha, 2012: 759-760 (fig. 5G-K).

Gastropoda, Costellariidae

Paratypes (2 spc): MZSP 102858.

Locality: Brazil, Espírito Santo, off Regência, 1940' S, 37²48' W, MD55 sta. CB77, 790-940 m depth, 27 May 1987.

Collectors: P. Bouchet, J.H. Leal and B. Métivier.

Preservation: Dry.

Remarks: Former MNHN, Paris.

\section{jukyriuva, Natica Simone, 2014}

Natica jukyriuva Simone, 2014: 582 (fig. 9A-E).

Gastropoda, Naticidae

Paratypes (2 spc): MZSP 105144.

Locality: Brazil, Espírito Santo, off Itaúnas, 1859' S, 3750' W, 295 m depth (type locality), 27 May 1987.

Collectors: P. Bouchet, J.H. Leal and B. Métivier.

Preservation: Dry.

Remarks: Former MNHN, Paris.

\section{lacrima, Pseudosimnia Simone \& Cunha, 2012}

Pseudosimnia lacrima Simone \& Cunha, 2012: 752-754 (fig. 3M-O).

Gastropoda, Ovulidae

Paratype: MZSP 104319.

Locality: Brazil, Espírito Santo, off Regência, 1940' S, 37048' W, MD55 sta. CB77, 790-940 m depth, 27 May 1987. 
Collectors: P. Bouchet, J.H. Leal and B. Métivier.

Preservation: Dry.

Remarks: Former MNHN, Paris.

lapernoi, Yoldiella Benaim \& Absalão, 2011

Yoldiella lapernoi Benaim \& Absalão, 2011: 515-517 (fig. 2A-G, J).

Bivalvia, Yoldiidae

Paratype (1 valve): MZSP 93356.

Locality: Brazil, Rio de Janeiro, Campos Basin, sta. 41, 22³9'34.36" S, 4008'22.27" W, 1200 m depth (type locality).

Collector: R/V Astro-Garoupa.

Preservation: Dry.

\section{latiusculus, Microlinices Simone, 2014}

Microlinices latiusculus Simone, 2014: 567-573 (figs 1A-K, 2A-H, 3A-E, 11D-E).

Gastropoda, Naticidae

Paratype: MZSP 105134.

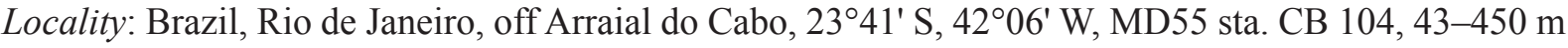
depth (type locality), 1 Jun. 1987.

Collectors: P. Bouchet, J.H. Leal and B. Métivier.

Preservation: Dry.

Remarks: Former MNHN, Paris.

\section{lepta, Peristichia Pimenta, Santos \& Absalão, 2008}

Peristichia lepta Pimenta, Santos \& Absalão, 2008: 182 (fig. 5D-H).

Gastropoda, Pyramidellidae

Holotype: MZSP 77062.

Paratypes $^{1}(20$ spc): MZSP 77063.

Paratypes $^{2}$ (2 spc): MZSP 77064.

Localities: Brazil, off Paraná; REVIZEE sta. 6656, 25⒉ $22.1^{\prime} \mathrm{S}, 46^{\circ} 30.35^{\prime} \mathrm{W}, 70 \mathrm{~m}$ depth; 1) off São Paulo,

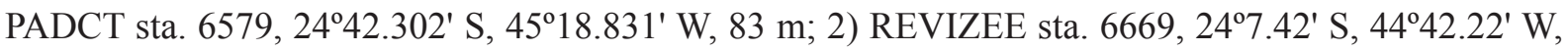
$101 \mathrm{~m}$.

Collector: R/V W. Besnard.

Preservation: Dry.

\section{litosbathron, Triptychus Pimenta, Santos \& Absalão, 2008}

Triptychus litosbathron Pimenta, Santos \& Absalão, 2008: 180 (fig. 4E-I).

Gastropoda, Pyramidellidae

Holotype: MZSP 77065.

Paratype $^{1}$ : MZSP 77066.

Paratype $^{2}$ : MZSP 86686.

Paratypes $^{3}$ (5 spc): MZSP 86688.

Paratype $^{4}$ : MZSP 86691.

Paratype $^{5}$ : MZSP 86693.

Paratype $^{6}$ : MZSP 86694.

Paratype $^{7}$ : MZSP 86695. 


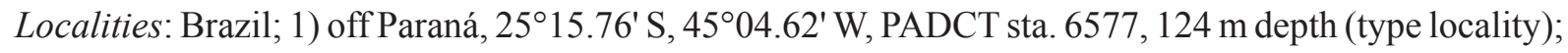
2) $24^{\circ} 40.75^{\prime} \mathrm{S}, 4^{\circ} 50.82^{\prime} \mathrm{W}$, REVIZEE sta. $6677,137 \mathrm{~m}$; 3) $24^{\circ} 00.95^{\prime} \mathrm{S}, 43^{\circ} 55.54^{\prime} \mathrm{W}$, REVIZEE sta.

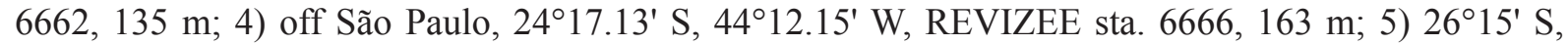
455' W, PADCT sta. 6641, $130 \mathrm{~m}$; 6) $26^{\circ} 17.134^{\prime} \mathrm{S}, 46^{\circ} 41.788^{\prime} \mathrm{W}$, REVIZEE sta. 6695, $153 \mathrm{~m}$; 7) $27^{\circ} 48.07^{\prime} \mathrm{S}, 47^{\circ} 24.04^{\prime} \mathrm{W}$, PADCT sta. $6606,175 \mathrm{~m}$.

Preservation: Dry.

\section{lucianae, Flabellina engeli Dacosta, Cunha, Simone \& Schrödl, 2007}

Flabellina engeli lucianae Dacosta, Cunha, Simone \& Schrödl, 2007: 340-343 (figs 1-10).

Gastropoda, Flabellinidae

Holotype: MZSP 48251.

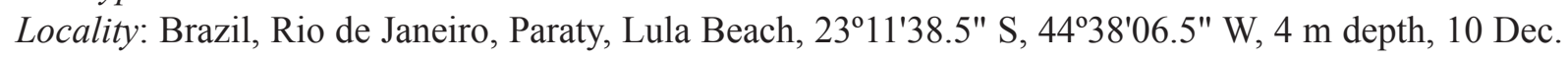
2005.

Collector: L.R. Simone.

Preservation: $70 \%$ ethanol.

macrostoma, Aclis Barros, Lima \& Francisco, 2007

Aclis macrostoma Barros, Lima \& Francisco, 2007: 62-63 (figs 1-6).

Gastropoda, Aclididae

Holotype: MZSP 77988.

Paratype: MZSP 77989.

Paratypes $^{2}$ (2 spc): MZSP 77990.

Localities: Brazil, off Rio Grande do Norte, continental slope off northeastern Brazil, muddy bottom,

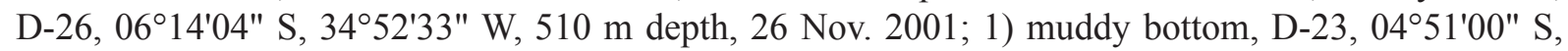
$35^{\circ} 06^{\prime} 46^{\prime \prime}$ W, 375 m, 24 Nov. 2001; 2) muddy bottom, D-27, 06²14'24" S, 3452'06" W, 500 m, 26 Nov. 2001.

Preservation: Dry.

\section{maculata, Oxychona Salvador \& Cavallari, 2013}

Oxychona maculata Salvador \& Cavallari, 2013a: 315-316 (figs 2-11).

Gastropoda, Orthalicidae

Holotype: MZSP 108005.

Paratype: MZSP 74500.

Localities: Brazil, Bahia, Ilhéus Municipality, $14^{\circ} 47^{\prime} \mathrm{S}, 39^{\circ} 2^{\prime} \mathrm{W}$, Dec. 1994; 1) Itapetinga Municipality, $15^{\circ} 15^{\prime}$ S, 40¹5' W, Jan. 2007.

Collectors: A. Bodart; 1) C. Lima.

Preservation: Dry.

\section{mangle, Crassostrea Amaral \& Simone, 2014}

Crassostrea mangle Amaral \& Simone, 2014: 2-6 (figs 1-2).

Bivalvia, Ostreidae

Holotype: MZSP 89462.

Paratypes $^{1}$ (40 spc): MZSP 100495.

Locality: Brazil, Alagoas State, Barra de Camaragibe, Camaragibe River Estuary (type locality), 21 Oct. 2008; 1) 8 Jan. 2011.

Collector: M.D.S. Tavares.

Preservation: $70 \%$ ethanol. 


\section{mariaodeteae, Poremskiconus Petuch \& Myers, 2014}

Poremskiconus mariaodeteae Petuch \& Myers, 2014b: 33 (text fig. + fig. 2A-F).

Gastropoda, Conidae

Holotype: MZSP 116525.

Paratype: MZSP 116526.

Locality: Brazil, Ceará state, off Camocim, $40 \mathrm{~m}$ depth.

Collector: D. Monteiro.

Preservation: Dry.

Remarks: This species was recombined as Conus mariaodeteae by Puillandre et al. (2015).

\section{marinae, Jaspidiconus Petuch \& Myers, 2014}

Jaspidiconus marinae Petuch \& Myers, 2014b: 35-38 (text fig. + fig. 4A-F).

Gastropoda, Conidae

Holotype: MZSP 116530.

Paratype: MZSP 116531.

Locality: Brazil, Bahia, Salvador, Todos os Santos Bay, northern coast of Itaparica Island, off Porto de Itaparica, $1 \mathrm{~m}$ depth.

Collector: G.S.P. Oliveira.

Preservation: Dry.

Remarks: This species was recombined as Conasprella marinae by Puillandre et al. (2015).

metamorphosicum, Caecum Lima, Santos \& Absalão, 2013

Caecum metamorphosicum Lima, Santos \& Absalão, 2013: 769-773 (figs 3-6, table 1).

Gastropoda, Caecidae

Paratypes (9 spc): MZSP 110294.

Paratypes $^{1}$ (6 spc): MZSP 110299.

Paratypes $^{2}$ (8 spc): MZSP 110308.

Paratypes $^{3}$ (1 spc): MZSP 110310.

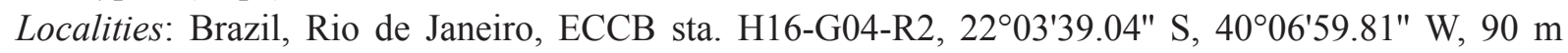

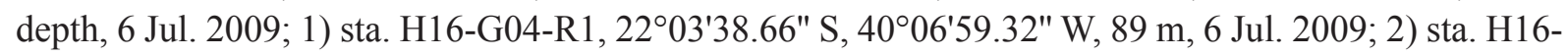

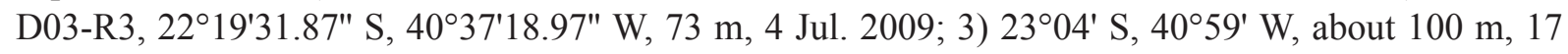
Dec. 2004.

Collector: R/V Seward Johnson.

Preservation: Dry.

\section{microsculpta, Hubendickia Brandt, 1968}

Hubendickia microsculpta Brandt, 1968: 239-240 (pl. 9, fig. 27 + text fig. 17).

Gastropoda, Pomatiopsidae

Probable paratype: MZSP 95945 (Fig. 4G-H).

Locality: East Thailand, Ubon Province, Mekong River at Bandan (type locality), 9 May 1967.

Preservation: Dry.

Remarks: Former Jens Hemmen Collection. Data on the specimen's label correspond to the lot originally catalogued as SMRL 3378. The specimen listed above is a possible remainder of a 50-specimen paratype lot left by the author under the care of the SMRL. This species was recombined as Manningiella microsculpta by Brandt (1974). 
Cratena minor Padula, Araújo, Matthews-Cascón \& Schrödl, 2014: 581-582 (figs 3B, 4A, D, 5A).

Gastropoda, Facelinidae

Paratype: MZSP 116702.

Locality: Brazil, Pernambuco State, Ponta Itapessoca, 15 Mar. 2005.

Collector: M. Schrödl.

Preservation: $99 \%$ ethanol.

mirim, Acteon Cunha, 2011

Acteon mirim Cunha, 2011: 230-231 (figs 2-8).

Gastropoda, Acteonidae

Holotype: MZSP 70344.

Paratype: MZSP 57091.

Paratype: MZSP 57092.

Paratype: MZSP 94212.

Locality: Brazil, Ceará, off Fortaleza, Canopus Bank, 02¹4'25" S, 38²2'50" W, 240 m depth, dredged, Aug.-Sep. 2005.

Collectors: J. Coltro and P.M. Costa.

Preservation: Dry.

mirim, Mitromorpha Simone \& Cunha, 2012

Mitromorpha mirim Simone \& Cunha, 2012: 771-772 (fig. 9G-K).

Gastropoda, Mitromorphidae

Paratype: MZSP 102674.

Paratype 1 : MZSP 102683.

Localities: Brazil, Espírito Santo, off Itaúnas, Abrolhos Slope, 1856' S, 3757' W, MD55 sta. DC82, 85-105 m depth; 1) off Itaúnas, $18^{\circ} 49^{\prime}$ S, 37 $7^{\circ} 57^{\prime} \mathrm{W}$, MD55 sta. DC83, 60 m; all collected on 28 May 1987.

Collectors: P. Bouchet, J.H. Leal and B. Métivier.

Preservation: Dry.

Remarks: Former MNHN, Paris.

\section{mohicanus, Cyclostremiscus Simone, 2012}

Cyclostremiscus mohicanus Simone, 2012c: 78-79 (figs 1-15).

Gastropoda, Tornidae

Holotype: MZSP 106551.

Paratypes (16 spc): MZSP 106552.

Locality: Brazil, Espírito Santo, São Mateus, off Guriri, $18^{\circ} 47^{\prime}$ S, 32 $32^{\circ} 39^{\prime}$ W, 3 m depth (type locality). Preservation: $70 \%$ ethanol. Paratypes are preserved dry, and a single specimen bears a metallic coating due to SEM examination.

molaris, Streptartemon Simone \& Casati, 2013

Streptartemon molaris Simone \& Casati, 2013: 154-156 (figs 43-48).

Gastropoda, Streptaxidae

Holotype: MZSP 112451.

Paratype $^{1}$ : MZSP 111840. 
Paratypes $^{2}$ (4 spc): MZSP 111841.

Paratype $^{3}$ : MZSP 111843.

Paratype $^{4}$ : MZSP 112366.

Paratypes $^{5}$ (2 spc): MZSP 112367.

Paratype $^{6}$ : MZSP 112371.

Paratype $^{7}$ : MZSP 112376.

Localities: Brazil, Piauí, Serra da Capivara, Coronel José Dias Municipality, close to Sítio do Mocó Town, Toca de Cima dos Pilão Cave, 851'47.10" S, 42³3'26.96" W; 1) same, sta. P2; 2) same, sta. P4; 3) same, sta. P2; all collected in 2012; 4-7) from type locality.

Collector: R. Casati.

Preservation: Dry.

\section{munensis, Hydrorissoia Brandt, 1968}

Hydrorissoia munensis Brandt, 1968: 243 (pl. 9, fig. $30+$ text fig. 21).

Gastropoda, Pomatiopsidae

Probable paratype: MZSP 95929 (Fig. 3K-L).

Locality: East Thailand, Ubon Province, Mun River at Phibun Mangsahan (type locality), 21 Jan. 1966. Preservation: Dry.

Remarks: Former Jens Hemmen Collection. Data on the specimen's label correspond to the lot originally catalogued as SMRL 3471. The specimen listed above is a possible remainder of a 50-specimen paratype lot left under the care of the SMRL. This species is a synonym of Hydrorissoia gracilis Brandt, 1968 according to Davis (1979).

\section{munensis, Jullienia Brandt, 1974}

Jullienia munensis Brandt, 1974: 83 (pl. 6, fig. 1).

Gastropoda, Pomatiopsidae

Probable paratopotype: MZSP 95482 (Fig. 4A-B).

Locality: Thailand, Mangsahan, Mun River at Phibun (type locality), 21 Jan. 1966.

Preservation: Dry.

Remarks: Former Jens Hemmen Collection. Measurements and label data compare fittingly with a 200-specimen paratype lot from the same locality, originally catalogued as SMRL 3461.

\section{neglecta, Angaria Poppe \& Goto, 1993}

Angaria neglecta Poppe \& Goto, 1993: 22 (pl. 6).

Gastropoda, Angariidae

Paratype: MZSP 95951.

Locality: Japan, Wakayama prefecture, Nada-Cho, $510 \mathrm{fms}$.

Preservation: Dry.

Remarks: Former Jens Hemmen Collection. The specimen listed above was initially designated as paratype \#12 in the original description. The senior author (Poppe, pers. comm.) confirmed the type status.

\section{nivea, Steeriana Nordsieck, 2005}

Steeriana nivea Nordsieck, 2005b: 211 (fig. 16).

Gastropoda, Clausiliidae

Paratype: MZSP 95479 (Fig. 6G-H). 
Locality: Peru, La Libertad Department, c. $25 \mathrm{~km}$ from Cajabamba to Huamachuco, c. $3200 \mathrm{~m}$ altitude (type locality), 4 Apr. 2012.

Collector: J. Hemmen.

Preservation: Dry.

Remarks: Former Jens Hemmen Collection. The specimen listed above is a remainder of a 21-specimen paratype lot deposited by Nordsieck in Hemmen's collection.

\section{notialis, Oscilla Pimenta, Santos \& Absalão, 2008}

Oscilla notialis Pimenta, Santos \& Absalão, 2008: 175-176 (fig. 2A-F).

Gastropoda, Pyramidellidae

Paratype 1 : MZSP 86787.

Paratypes $^{2}$ (5 spc): MZSP 86788.

Paratypes $^{3}$ (2 spc): MZSP 86790.

Paratypes $^{4}$ (5 spc): MZSP 86791.

Paratypes $^{5}$ (29 spc): MZSP 86792.

Paratype $^{6}$ : MZSP 86793.

Paratypes $^{7}$ (14 spc): MZSP 86794.

Paratypes $^{8}$ (2 spc): MZSP 86795.

Paratypes $^{9}$ (9 spc): MZSP 86798.

Paratypes $^{10}$ (11 spc): MZSP 86799.

Paratype $^{11}$ : MZSP 86800.

Paratype $^{12}$ : MZSP 86801.

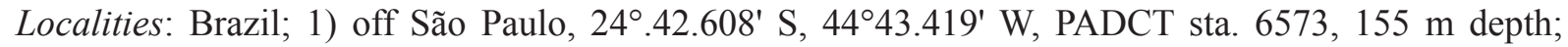
2) $26^{\circ} 15^{\prime} \mathrm{S}, 45^{\circ} 53^{\prime} \mathrm{W}$, PADCT sta. $6541,130 \mathrm{~m}$; 3) $25^{\circ} 46^{\prime} \mathrm{S}, 4^{\circ} 28.8^{\prime} \mathrm{W}$, PADCT sta. $6631,164 \mathrm{~m}$; 4) off Rio de Janeiro, $21^{\circ} 51.6^{\prime} \mathrm{S}, 47^{\circ} 42^{\prime} \mathrm{W}$, PADCT sta. 6617, $327 \mathrm{~m}$; 5) off São Paulo, $24^{\circ} 12.74^{\prime} \mathrm{S}$,

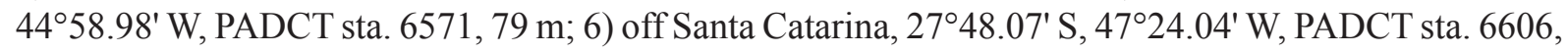
175 m depth; 7) off São Paulo, $25^{\circ} 15.76^{\prime}$ S, $45^{\circ} 04.62^{\prime}$ W, PADCT sta. 6577, 124 m; 8) off Santa Catarina,

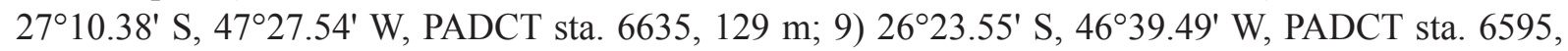

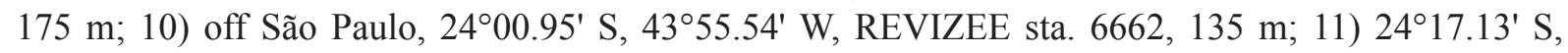
$44^{\circ} 12.15^{\prime}$ W, REVIZEE sta. 666, $163 \mathrm{~m}$; 12) off Santa Catarina, $26^{\circ} 15^{\prime} \mathrm{S}, 45^{\circ} 53^{\prime} \mathrm{W}$, PADCT sta. 6641 , $153 \mathrm{~m}$.

Collector: R/V W. Besnard.

Preservation: Dry.

\section{notialis, Solemya Simone, 2009}

Solemya notialis Simone, 2009: 423-434 (figs 39-46, 111-117).

Bivalvia, Solemyidae

Holotype: MZSP 88440.

Paratype: MZSP 20935.

Paratypes $^{1}$ ( 2 spc): MZSP 20936.

Paratype 2 : MZSP 20937.

Paratype $^{3}$ : MZSP 35257.

Localities: Brazil, São Paulo, off Ubatuba, sta. $12,2^{\circ} 25^{\prime} \mathrm{S}, 44^{\circ} 43^{\prime} \mathrm{W}, 33.5 \mathrm{~m}$ depth (type locality), 17 Dec. 1965; 1) Rio de Janeiro, Angra dos Reis, sta 53, 31 m, 29 Jun. 1966; 2) sta. 54, 27 m, 29 Jun. 1966; 3) Cabo Frio, May 2002.

Collectors: R/V Emilia; 3) P. Gonçalves.

Preservation: Dry and 70\% ethanol. 


\section{nymphalis, Cranopsis Simone \& Cunha, 2014}

Cranopsis nymphalis Simone \& Cunha, 2014: 443-444 (figs 31-39).

Gastropoda, Fissurellidae

Paratypes (14 spc): MZSP 102927.

Paratypes (19 spc): MZSP 107717.

Locality: Brazil, Espírito Santo, off Itaúnas, Abrolhos Slope, 1859' S, 3750' W, 295 m depth, MD55 sta. DC75 (type locality), 27 May 1987.

Collectors: P. Bouchet, J.H. Leal \& B. Métivier.

Preservation: Dry.

Remarks: Former MNHN, Paris.

onager, Bulimus Beck, 1837

Bulimus (Bulimulus) onager Beck, 1837: 64.

Gastropoda, Orthalicidae

Neotype: MZSP 29794.

Locality: Brazil, Bahia, Itabuna, city center coordinates $14^{\circ} 47^{\prime} 09^{\prime \prime} \mathrm{S}, 39^{\circ} 16^{\prime} 48^{\prime \prime} \mathrm{W}, \sim 54 \mathrm{~m}$ altitude, Aug. 1900.

Collector: E. Garbe.

Preservation: Dry.

Remarks: Designated by Salvador \& Cavallari (2013b). For additional information, see Salvador et al. (2014a).

onca, Isotriphora Fernandes, Pimenta \& Leal, 2013

Isotriphora onca Fernandes, Pimenta \& Leal, 2013: 10-11 (figs 9-10, 45-50).

Gastropoda, Triphoridae

Paratypes (2 spc): MZSP 112068.

Locality: Brazil, Vitória-Trindade Chain, Trindade Island, REVIZEE sta. C5-41F, 20³0' S, 29¹6' W, $360 \mathrm{~m}$ depth (type locality).

Collector: REVIZEE-Central.

Preservation: Dry.

ogum, Jaspidiconus Petuch \& Myers, 2014

Jaspidiconus ogum Petuch \& Myers, 2014a: 44-45 (fig. 3H-I).

Gastropoda, Conidae

Holotype: MZSP 116145.

Locality: Brazil, Bahia State, Itaparica Island, off Aratuba, 2 m depth.

Preservation: Dry.

Remarks: This species was recombined as Conasprella ogum by Puillandre et al. (2015).

\section{ovir, Turricostellaria Simone \& Cunha, 2012}

Turricostellaria ovir Simone \& Cunha, 2012: 760-762 (fig. 6A-D).

Gastropoda, Costellariidae

Paratypes (2 spc): MZSP 102860.

Localities: Brazil, Espírito Santo, off Conceicão da Barra, 1859' S, 3747' W, MD55 sta. CB70, 1540 1550 m; 26-27 May 1987.

Collectors: P. Bouchet, J.H. Leal and B. Métivier. 
Preservation: Dry.

Remarks: Former MNHN, Paris.

panhai, Arinia Maassen, 2001

Arinia panhai Maassen, 2001: 55-56 (fig. 4, 12-13).

Gastropoda, Diplommatinidae

Paratype: MZSP 95493 (Fig. 1C-D).

Locality: Thailand, Yala Province, Kransang Cave, c. 2.6 off km 45.7 rd\# 410 (Yala-Betong), 4 Oct. 2000.

Preservation: Dry.

Remarks: Former Jens Hemmen Collection. The specimen's label bears a paratype status annotation. It is a remainder of a 45-specimen paratype lot originally deposited in Hemmen's collection (Maassen 2001).

\section{paranapuaensis, Yoldiella Benaim \& Absalão, 2011}

Yoldiella paranapuaensis Benaim \& Absalão, 2011: 517-518 (fig. 3A-B, E-H).

Bivalvia, Yoldiidae

Paratype (1 valve): MZSP 93357.

Locality: Brazil, Rio de Janeiro, Campos Basin, 2244'49" S, 40¹0'06" W, sta. 66, 1323 m depth, 2003.

Collector: R/V Astro-Garoupa.

Preservation: Dry.

\section{patriceae, Lamniconus Petuch \& Myers, 2014}

Lamniconus patriceae Petuch \& Myers, 2014a: 39 (fig. 1F-H).

Gastropoda, Conidae

Holotype: MZSP 116142.

Locality: Brazil, Rio de Janeiro State, Macaé, $300 \mathrm{~m}$ depth.

Collectors: Shrimp boats.

Preservation: Dry.

Remarks: This species was recombined as Conus patriciae by Puillandre et al. (2015).

\section{petalius, Ovini Simone, 2013}

Ovini petalius Simone 2013a: 187-188 (figs A-J).

Gastropoda, Tornidae

Holotype: MZSP 107806.

Locality: Brazil, Espírito Santo, Guriri, $18^{\circ} 47^{\prime} \mathrm{S}, 32^{\circ} 39^{\prime} \mathrm{W}, 3 \mathrm{~m}$ depth.

Preservation: Dry, with a metallic coating (SEM).

\section{pingius, Dolichupis Simone \& Cunha, 2012}

Dolichupis pingius Simone \& Cunha, 2012: 756-757 (fig. 4E-H).

Gastropoda, Triviidae

Paratypes (2 spc): MZSP 104030.

Locality: Brazil, Espírito Santo, off Regência, Abrolhos Slope, 1940' S, 37²48' W, MD55 sta. CB77, 790-940 m depth, 27 May 1987.

Collectors: P. Bouchet, J.H. Leal and B. Métivier.

Preservation: Dry.

Remarks: Former MNHN, Paris. 


\section{pipoca, Natica Simone, 2014}

Natica pipoca Simone, 2014: 582-583 (fig. 9F-I).

Gastropoda, Naticidae

Paratypes (2 spc): MZSP 105144.

Locality: Brazil, Espírito Santo, off Itaúnas, 1859' S, 3750' W, 295 m depth (type locality), 27 May 1987.

Collectors: P. Bouchet, J.H. Leal and B. Métivier.

Preservation: Dry.

Remarks: Former MNHN, Paris.

polita, Manningiella Brandt, 1970

Manningiella polita Brandt, 1970: 195-196 (pl. 13, fig. 13 + text fig. 13).

Gastropoda, Pomatiopsidae

Probable paratype: MZSP 95475 (Fig. 4I-J).

Locality: Thailand, Ubon Province, in the mouth of Mun River at Ban dan Kao, 24 Apr. 1969.

Preservation: Dry.

Remarks: Former Jens Hemmen Collection. Measurements and data on the specimen's label correspond to the lot originally catalogued as SMRL 16361 . The specimen listed above is a possible remainder of a 2000-specimen paratype lot from the above-mentioned locality. According to Brandt (1970), these specimens were left under the care of the SMRL.

\section{pongoensis, Cylindronenia pangamitoensis Nordsieck, 2005}

Cylindronenia pangamitoensis pongoensis Nordsieck, 2005b: 209-210 (fig. 13).

Gastropoda, Clausiliidae

Paratype: MZSP 95470 (Fig. 6C-D).

Locality: Peru, Amazonas Department, Pongo de Rentema, Bagua Chica (type locality), area with bushes near large rocky walls, Dec. 1998.

Preservation: Dry.

Remarks: Former Jens Hemmen Collection. The specimen listed above is a remainder of an 8-specimen paratype lot deposited by Nordsieck in Hemmen's collection.

\section{pomponeti, Jaspidiconus Petuch \& Myers, 2014}

Jaspidiconus pomponeti Petuch \& Myers, 2014b: 38-39 (text fig. + fig. 4G-J).

Gastropoda, Conidae

Holotype: MZSP 116532.

Paratype: MZSP 116533.

Locality: Brazil, Bahia State, Salvador, Todos os Santos Bay, off Ribeira, 0.5 m depth.

Collector: G.S.P. Oliveira.

Preservation: Dry.

Remarks: This species was recombined as Conasprella pomponeti by Puillandre et al. (2015).

\section{poremskii, Jaspidiconus Petuch \& Myers, 2014}

Jaspidiconus poremskii Petuch \& Myers, 2014a: 45 (fig. 3D-E).

Gastropoda, Conidae

Holotype: MZSP 116145.

Locality: Brazil, Bahia State, Corumbau, near the reef, $1 \mathrm{~m}$ depth. 
Collector: A. Poremskii.

Preservation: Dry.

Remarks: This species was recombined as Conasprella poremskii by Puillandre et al. (2015).

\section{praia, Ostrea parasitica Ihering, 1907}

Ostrea parasitica praia Ihering, 1907: 426.

Bivalvia, Ostreidae

Syntype (2 spc): MZSP 10916.

Locality: Brazil, Rio Grande do Sul State, 1883.

Collector: H. Ihering.

Preservation: Dry.

Remarks: Detailed information on the taxonomy, as well as figures of this species, can be found in Amaral \& Simone (2014).

\section{priscus, Habeas Simone, 2013}

Habeas priscus Simone, 2013b: 522 (figs 11-17).

Gastropoda, Diplommatinidae

Holotype: MZSP 103044.

Locality: Brazil, Bahia, Central, Boqueirão do Maxixe, sta. LES0317, 1346'51.6" S, 4402'18.7" W, 26 Jan. 2007.

Collectors: M.E. Bichuette and F.D. Passos.

Preservation: Dry.

\section{prolatus, Anctus Simone \& Casati, 2013}

Anctus prolatus Simone \& Casati, 2013: 151-153 (figs 35-41).

Gastropoda, Odontostomidae

Holotype: MZSP 112450.

Paratypes $^{1}$ (3 spc): MZSP 111836.

Parartype $^{2}$ : MZSP 112350.

Paratype $^{3}$ (2 spc): MZSP 112362.

Paratype $^{4}(3$ spc): MZSP 112372.

Localities: Brazil, Piauí, Serra da Capivara, Coronel José Dias Municipality, close to Sítio do Mocó Town, Toca de Cima dos Pilão Cave, 851'47.10" S, 42³3'26.96" W; 1) sta. P6; all collected in 2012. Collector: R. Casati.

Preservation: Dry.

punctata, Spiripockia Simone, 2012

Spiripockia punctata Simone, 2012a: 516-523 (figs 1-33).

Gastropoda, Pomatiopsidae

Holotype: MZSP 105000.

Paratypes (19 spc): MZSP 104435.

Locality: Brazil, Bahia, Serra Ramalho Karst Area, Middle São Francisco River Basin, Lapa dos Peixes Cave, 1349'21.78" S, 4357'24.39" W (type locality) 29 Jul. 2005.

Collector: M.E. Bichuette.

Preservation: Dry, $70 \%$ ethanol. 


\section{pussa, Zeidora Simone \& Cunha, 2014}

Zeidora pussa Simone \& Cunha, 2014: 460-461 (figs 125-132).

Gastropoda, Fissurellidae

Paratypes (2 spc): MZSP 105510.

Paratype 1 : MZSP 105511.

Paratype $^{2}$ : MZSP 107728.

Localities: Brazil, Espírito Santo, off Itaúnas, 1859' S, 3750’ W, MD55 sta. DC75, 295 m depth, May 1987; 1) off Regência, 1940' S, 3744' W, MD55 sta. CB77, 790-940 m, May 1987; 2) off Conceição da Barra, Abrolhos Slope, $18^{\circ} 59^{\prime}$ S, 3750' W, MD55 sta. CB76, 637 m (type locality).

Collectors: P. Bouchet, J.H. Leal and B. Métivier.

Preservation: Dry.

Remarks: Former MNHN, Paris.

\section{quinni, Solariella Barros \& Pereira in Barros, Santos \& Francisco 2008}

Solariella quinni Barros \& Pereira in Barros, Santos, \& Francisco, 2008: 13-14 (figs 15-21).

Gastropoda, Solariellidae

Holotype: MZSP 77083.

Paratype: MZSP 77082.

Paratype 2 : MZSP 77084.

Paratype: MZSP 77085.

Localities: Brazil, Pernambuco, 08 09' S, 3434' W, gravel bottom, 69-71 m depth, 19 Dec. 2004; 1) $08^{\circ} 11^{\prime} \mathrm{S}, 34^{\circ} 34^{\prime} \mathrm{W}, 66-71 \mathrm{~m}, 18$ Dec. 2004 ; 2) $08^{\circ} 11^{\prime} \mathrm{S}, 34^{\circ} 36^{\prime} \mathrm{W}, 69-71 \mathrm{~m}, 19$ Dec. 2004; 3) $08^{\circ} 11^{\prime} \mathrm{S}$, 34³6' W, 69-71 m, 19 Dec. 2004.

Preservation: Dry.

\section{regimaensis, Trochoidea Brandt, 1959}

Trochoidea (Xeroregima) regimaensis Brandt, 1959: 99-101 (pl. 6, fig. 13 + text fig. 8).

Gastropoda, Hygromiidae

Probable paratype: MZSP 95910 (Fig. 6L-N).

Locality: Libya, Cyrenaika, Slonta (on track to Chadra), 10 Nov. 1957.

Collector: R. Brandt.

Preservation: Dry.

Remarks: Former Jens Hemmen Collection. Data on the specimen's label and measurements compare well with an 1140-specimen paratype lot originally catalogued as C837.

\section{rolfbrandti, Hubendickia Temcharoen, 1971}

Hubendickia rolfbrandti Temcharoen, 1971: 96-98 (pl. 6, fig. 6 + text fig. 6).

Gastropoda, Pomatiopsidae

Probable paratype: MZSP 95948 (Fig. 3E-F).

Locality: Laos, Mekong River, Muang Khong, near Wat (type locality), 20 Mar. 1969.

Preservation: Dry.

Remarks: Former Jens Hemmen Collection. As per measurements and data on the specimens' label, this is a possible remainder of a 1000-specimen paratype lot originally catalogued as SMRL 16076. According to Temcharoen (1971), these specimens were left under the care of the SMRL. 


\section{rolfbrandti, Jullienia Temcharoen, 1971}

Jullienia rolfbrandti Temcharoen, 1971: 99-100 (pl. 6, fig. $9+$ text fig. 9).

Gastropoda, Pomatiopsidae

Probable paratype: MZSP 95463 (Fig. 4C-D).

Locality: Laos, Mekong River at Muang Khong, near Wat (type locality), 22 Feb. 1969.

Preservation: Dry.

Remarks: Former Jens Hemmen Collection. As per measurements and data on the specimens' label, this is a possible remainder of a 100-specimen paratype lot originally catalogued as SMRL 16326. According to Temcharoen (1971), these specimens were left under the care of the SMRL.

\section{rolfbrandti, Manningiella Temcharoen, 1971}

Manningiella rolfbrandti Temcharoen, 1971: 98-99 (pl. 6, fig. $8+$ text fig. 7).

Gastropoda, Pomatiopsidae

Probable paratype: MZSP 95924 (Fig. 4K-L).

Locality: Laos, Mekong River, Muang Khong, near Wat (type locality), 22 Feb. 1969.

Preservation: Dry.

Remarks: Former Jens Hemmen Collection. As per measurements and data on the specimens' label, this is a possible remainder of a 100-specimen paratype lot originally catalogued as SMRL 16287. According to Temcharoen (1971), these specimens were left under the care of the SMRL.

\section{rudigerbieleri, Solatisonax Tenório, Barros, Francisco \& Silva, 2011}

Solatisonax rudigerbieleri Tenório, Barros, Francisco \& Silva, 2011: 175-178 (figs 1A-C, E-F).

Gastropoda, Architectonicidae

Paratype: MZSP 85951.

Paratype': MZSP 85952.

Localities: Brazil, off Pernambuco, 06²5.6' S, 3443.9' W, D-01, muddy bottom, 425 m, 29 Aug. 1999; 1) $08^{\circ} 46.5^{\prime} \mathrm{S}, 34^{\circ} 44.5^{\prime} \mathrm{W}$, D-11, muddy bottom, $690 \mathrm{~m}$ (type locality), 18 Nov. 2000.

Preservation: Dry.

Remarks: Paratypes MZSP 85951 and MZSP 85952 correspond respectively to "paratype 2, MZUSP 15642" and "paratype 6, MZUSP 15643" reported in the original description by Tenório et al. (2011: 177). The catalogue numbers erroneously given by the authors (MZSP 15642 and MZSP 15643) are actually non-type lots belonging to different species/mollusc groups registered in the 1980's, and must be disregarded.

\section{sama, Mitromorpha Simone \& Cunha, 2012}

Mitromorpha sama Simone \& Cunha, 2012: 770-771 (fig. 9A-F).

Gastropoda, Mitromorphidae

Paratypes (2 spc): MZSP 102673.

Locality: Brazil, Espírito Santo, off Itaúnas, Abrolhos Slope, 1900' S, 37²4' W, MD55 sta. DC73, 602-620 m depth, 27 May 1987.

Collectors: P. Bouchet, J.H. Leal and B. Métivier.

Preservation: Dry.

Remarks: The specimens listed above were referred to as MZSP 102973 in the original description. This was merely a typographical error. Former MNHN, Paris. 
sanpaulensis, Loligo Brakoniecki, 1984

Loligo sanpaulensis Brakoniecki, 1984: 441-446 (figs 3-4).

Cephalopoda, Loliginidae

Paratype: MZSP 18498.

Paratype: MZSP 18499.

Localities: Brazil, Rio Grande do Sul, off Chuí, 34²0' S, 174 m depth, Nov. 1974.

Preservation: $70 \%$ ethanol.

Remarks: The specimens listed above were confirmed as types after the previous compilation (Dornellas \& Simone 2011). Brakoniecki (1984) attributed two catalogue numbers (MZSP 18498, MZSP 18499) for a single specimen in the original description. In a subsequent paper on the MZSP cephalopod collection, Perez \& Haimovici (1991) reported another lot as a paratype (MZSP 18601). However, Brakoniecki (1984) originally listed this lot as "other material", therefore not formally designating it as a paratype. This species was recombined as Doryteuthis sanpaulensis by Vecchione et al. (1998).

\section{santosi, Mitromorpha Lima, Barros \& Francisco, 2010}

Mitromorpha santosi Lima, Barros \& Francisco, 2010: 600 (fig. 1A-H).

Gastropoda, Mitromorphidae

Holotype: MZSP 78935.

Paratype: MZSP 78936.

Localities: Brazil, off Rio Grande do Norte, sta. 23, 04'51'40" S, 35066'01" W, 384 m depth, 24 Nov. 2001; 1) sta. 22, 04 $50^{\circ} 60^{\prime \prime}$ S, 3506'46" W, 375 m, 24 Nov. 2001.

Collector: R/V Natureza.

Preservation: Dry.

\section{schuetti, Paraprososthenia Brandt, 1968}

Paraprososthenia schuetti Brandt, 1968: 232 (pl. 8, fig. 17).

Gastropoda, Pomatiopsidae

Probable paratype: MZSP 95487 (Fig. 3G-H).

Locality: Thailand, Ubon Province, Mekong River at Bandan (type locality), 21 Mar. 1967.

Preservation: Dry.

Remarks: Former Jens Hemmen Collection. Measurements and data on the specimen's label correspond to the lot originally catalogued as SMRL 3382. The specimen listed above is a possible remainder of a 10-specimen paratype lot left under the care of the SMRL. This species was recombined as Hubendickia schuetti in Brandt (1974).

\section{scymnocelata, Boonea Pimenta, Absalão \& Miyaji, 2009}

Boonea scymnocelata Pimenta, Absalão \& Miyaji, 2009: 48-49 (figs 15-20).

Gastropoda, Pyramidellidae

Holotype: MZSP 89107.

Paratypes (2 spc): MZSP 89108.

Paratypes $^{1}$ (6 spc): MZSP 89109.

Paratype 2 : MZSP 89110.

Paratype: MZSP 89111.

Paratype $^{4}$ : MZSP 89112.

Paratype $^{5}$ : MZSP 89113.

Paratypes $^{6}$ (4 spc): MZSP 89114.

Paratype ${ }^{7}$ : MZSP 89115. 
Paratype ${ }^{8}$ : MZSP 89116.

Localities: Brazil, off Paraná, 26033.55' S, 46 $39.49^{\prime}$ W, PADCT sta. 6595, 175 m depth (type locality);

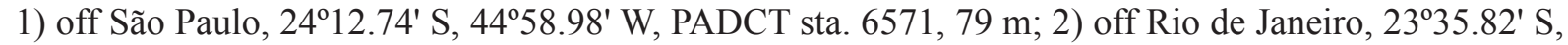

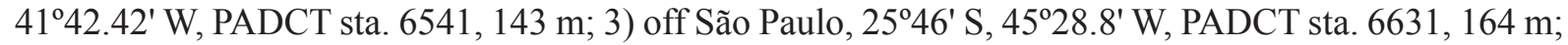

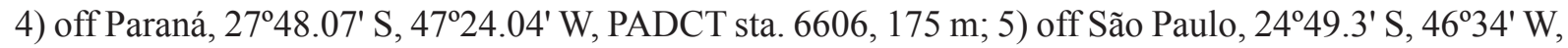
PADCT sta. 6642, $128 \mathrm{~m}$; 6) $25^{\circ} 46^{\prime} \mathrm{S}, 45^{\circ} 28.8^{\prime} \mathrm{W}$, PADCT sta. 6631, $164 \mathrm{~m}$; 7) $24^{\circ} 17.13^{\prime} \mathrm{S}, 44^{\circ} 12.15^{\prime} \mathrm{W}$,

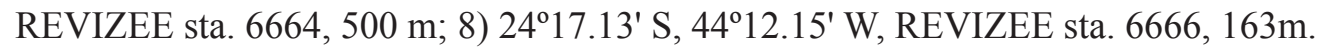

Collector: R/V W. Besnard.

Preservation: Dry.

secretum, Opisthostoma Maassen, 2002

Opisthostoma secretum Maassen, 2002: 179 (figs 45-46).

Gastropoda, Diplommatinidae

Probable paratype: MZSP 95917 (Fig. 2C-D).

Locality: Indonesia, West Sumatra, east of Payakumbuh, Kampung Desa Gadut, 00²15'35.1" S, 10044'01.1" E, Jul. 1997.

Collector: W.J.M. Maassen.

Preservation: Dry.

Remarks: Former Jens Hemmen Collection. Data on the specimen's label correspond to a 20 -specimen lot originally deposited in Maassen's reference collection.

selmaniana, Cernuella Brandt, 1959

Cernuella (Cernuella) selmaniana Brandt, 1959: 88-89 (pl. 6, fig. 4 + text fig. 2).

Gastropoda, Hygromiidae

Probable paratype: MZSP 95484 (Fig. 6I-K).

Locality: Libya, Cyrenaica, Ain es Selmani near Benghazi (type locality), 12 Jan. 1958.

Collector: R. Brandt.

Preservation: Dry.

Remarks: Former Jens Hemmen Collection. Data on the label and measurements compare fittingly with a 520-specimen paratype lot originally deposited in Brandt's reference collection.

\section{seraphina, Oenopota Figueira \& Absalão, 2010}

Oenopota seraphina Figueira \& Absalão, 2010b: 473-475 (fig. 1A-C).

Gastropoda, Mangeliidae

Paratypes (5 spc): MZSP 90704.

Paratypes (4 spc): MZSP 90705.

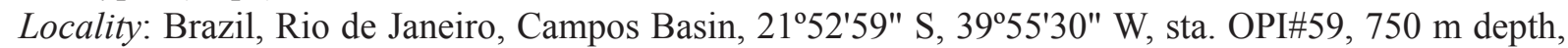
12 Dec. 2002.

Collector: R/V Astro Garoupa.

Preservation: Dry.

\section{siamensis, Hubendickia Brandt, 1968}

Hubendickia siamensis Brandt, 1968: 236-237 (pl. 8, fig. $22+$ text fig. 15).

Gastropoda, Pomatiopsidae

Paratype: MZSP 95466.

Locality: Thailand, Mun River at Phibun Mangsahan (type locality), 8 May 1966.

Preservation: Dry. 
Remarks: Former Jens Hemmen Collection. The specimen listed above is a possible remainder of several thousand specimens left under the care of the SMRL. This species is a junior synonym of Hubendickia sulcata (Bavay, 1895), according to Davis (1979).

\section{simonei, Jaspidiconus Petuch \& Myers, 2014}

Jaspidiconus simonei Petuch \& Myers, 2014a: 47 (fig. 3F-G).

Gastropoda, Conidae

Holotype: MZSP 116146.

Locality: Brazil, Rio de Janeiro State, Arraial do Cabo.

Preservation: Dry.

Remarks: This species was recombined as Conasprella simonei by Puillandre et al. (2015).

simonei, Terebra Lima, Tenório \& Barros, 2007

Terebra simonei Lima, Tenório \& Barros, 2007: 64-65 (figs 1-7).

Gastropoda, Terebridae

Holotype: MZSP 84234.

Paratype: MZSP 84235 (Fig. 5K-L).

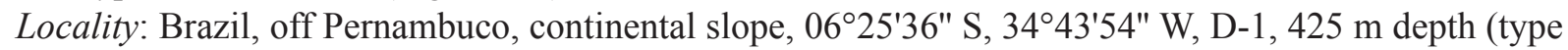
locality), 29 Aug. 1999.

Collector: R/V Natureza.

Preservation: Dry.

\section{strictecostata, Neniella Nordsieck, 2005}

Neniella strictecostata Nordsieck, 2005b: 207 (fig. 7).

Gastropoda, Clausiliidae

Paratype: MZSP 95947 (Fig. 6E-F).

Locality: Peru, Amazonas Department, c. $20 \mathrm{~km}$ from the bridge over Marañon River near Balsas to Leymebamba, $06^{\circ} 50.786^{\prime} \mathrm{S}, 7^{\circ} 57.728^{\prime} \mathrm{W}$ (type locality), on rocks, 2 Nov. 2004.

Collector: J. Hemmen.

Preservation: Dry.

Remarks: Former Jens Hemmen Collection. The specimen listed above is a remainder of an 8-specimen paratype lot deposited by Nordsieck in Hemmen's collection.

supralamellata, Diplommatina Maassen, 2007

Diplommatina supralamellata Maassen, 2007: 191-194 (figs 1-3).

Gastropoda, Diplommatinidae

Probable paratype: MZSP 95498 (Fig. 1I-J).

Locality: Indonesia, S Sulawesi, Tanah Toraja, $1.5 \mathrm{~km} \mathrm{~W}$ of Rantepao, 02 ${ }^{\circ} 57.18^{\prime} \mathrm{S}, 119^{\circ} 53.63^{\prime} \mathrm{E}, 750 \mathrm{~m}$ altitude, Jul. 2002.

Preservation: Dry.

Remarks: Former Jens Hemmen Collection. Data on the specimen's label correspond to a 25-specimen lot deposited in Maassen's reference collection, originally catalogued as MD 110.

\section{suspira, Emarginula Simone \& Cunha, 2014}

Emarginula suspira Simone \& Cunha, 2014: 453-455 (figs 90-102).

Gastropoda, Fissurellidae 
Paratype: MZSP 102921.

Paratypes $^{1}$ (5 spc): MZSP 102932.

Paratypes $^{2}$ (2 spc): MZSP 102939.

Localities: Brazil, Espírito Santo, off Regência, 1940' S, 3748' W, 790-940 m depth, MD55 sta. CB77; 1-2) off Itaúnas, Abrolhos Slope, 1859' S, 375' W, MD55 sta. DC75, 295 m (type locality), 27 May 1987.

Collectors: P. Bouchet, J.H. Leal and B. Métivier.

Preservation: Dry.

Remarks: Former MNHN, Paris.

\section{taludae, Paranadara Francisco, Barros \& Lima, 2012}

Paranadara taludae Francisco, Barros \& Lima, 2012: 1141-1142 (figs 1-6).

Bivalvia, Arcidae

Paratypes (2 spc): MZSP 84246.

Locality: Brazil, off Pernambuco, 'Natureza' sta. D-11, 0846' S, 3444' W, muddy bottom, $690 \mathrm{~m}$ depth, 18 Nov. 2000.

Collector: R/V Almirante Saldanha.

Preservation: Dry.

\section{tamandua, Cuspidaria Absalão \& Oliveira, 2011}

Cuspidaria tamandua Absalão \& Oliveira, 2011: 133-136 (figs 57-63).

Bivalvia, Cuspidariidae

Paratypes (2 valves): MZSP 96912.

Locality: Brazil, off Rio de Janeiro, Campos Basin, 22³7'02" S, 3956'20" W, 1950 m depth, 13 Jun. 2003.

Collector: R/V Astro Garoupa.

Preservation: Dry.

\section{tanata, Taranis Figueira \& Absalão, 2010}

Taranis tanata Figueira \& Absalão, 2010a: 736-737 (fig. 1G-I).

Gastropoda, Raphitomidae

Paratype: MZSP 90709.

Locality: Brazil, Rio de Janeiro, Campos Basin, 2204'45" S, 3941'58" W, sta. OPII\#53, 1910 m depth, 27 Jun. 2003.

Collector: R/V Astro Garoupa.

Preservation: Dry.

tessa, Anostoma Simone, 2012

Anostoma tessa Simone, 2012b: 435-436 (figs 15-20).

Gastropoda, Orthalicidae

Holotype: MZSP 103914.

Paratype: MZSP 103915.

Paratypes (37 spc): MZSP 103916.

Locality: Brazil, Bahia, Santa Maria da Vitória, $\sim 13^{\circ} 24^{\prime}$ S, 441' $\mathrm{W}, \sim 460$ m altitude (type locality), Jan. 2012.

Collector: J. Coltro.

Preservation: Dry. 


\section{tigrina, Isotriphora Fernandes, Pimenta \& Leal, 2013}

Isotriphora tigrina Fernandes, Pimenta \& Leal, 2013: 8-9 (figs 7-8, 39-44).

Gastropoda, Triphoridae

Paratypes (3 spc): MZSP 105154.

Locality: Brazil, Trindade Island, Vitória-Trindade Chain, $20^{\circ} 30^{\prime} \mathrm{S}, 29^{\circ} 19^{\prime} \mathrm{W}$, MD55 sta. DC59, $52-$ $60 \mathrm{~m}$ depth (type locality).

Collectors: P. Bouchet, J.H. Leal and B. Métivier.

Preservation: Dry.

\section{tonisii, Poremskiconus Petuch \& Myers, 2014}

Poremskiconus tonisii Petuch \& Myers, 2014b: 33-35 (text fig. + fig. 2G-H).

Gastropoda, Conidae

Holotype: MZSP 116527.

Locality: Brazil, Bahia, off Prado, Abrolhos Platform, 25 m depth.

Preservation: Dry.

Remarks: Recombined as Conus tonisii by Puillandre et al. (2015).

torquata, Eulimella Pimenta, Santos \& Absalão, 2011

Eulimella torquata Pimenta, Santos \& Absalão, 2011: 28-30 (figs 19-25).

Gastropoda, Pyramidellidae

Paratypes (2 spc): MZSP 101567.

Locality: Brazil, off Espírito Santo, 19²41' S, 37048' W, MD 55 sta. CB77, 27 May 1987.

Collectors: P. Bouchet, J.H. Leal and B. Métivier.

Preservation: Dry.

Remarks: Former MNHN, Paris.

\section{trindadense, Caecum Lima, Santos \& Absalão, 2013}

Caecum trindadense Lima, Santos \& Absalão, 2013: 773-776 (figs 7-10).

Gastropoda, Caecidae

Paratype: MZSP 110290.

Paratypes $^{1}$ (7 spc): MZSP 110292.

Paratypes $^{2}$ ( 5 spc): MZSP 110306.

Localities: Brazil, Rio de Janeiro, sta. D1, 22 $48^{\prime} \mathrm{S}, 41^{\circ} 09^{\prime} \mathrm{W}, 69 \mathrm{~m}$ depth, 23 Feb. 1996; 1) ECCB sta. H16-F03-R2, $22^{\circ} 07^{\prime} 43.33^{\prime \prime} \mathrm{S}, 40^{\circ} 18^{\prime} 48.95^{\prime \prime} \mathrm{W}, 71 \mathrm{~m}, 6$ Jul. 2009; 2) ECCB sta. H16-F03-R1, 22 $02^{\circ} 43.44^{\prime \prime}$ S, 40¹8'47.00" W, 72 m, 05 Jul. 2009.

Collectors: R/V Antares; 1-2) R/V Seward Johnson.

Preservation: Dry.

\section{trispiralis, Hydrorissoia Brandt, 1968}

Hydrorissoia trispiralis Brandt, 1968: 243-244 (pl. 9, fig. $31+$ text fig. 22).

Gastropoda, Pomatiopsidae

Topotype: MZSP 95936.

Locality: East Thailand, Ubon Province, Mekong River at Bandan (type locality), 22 Mar. 1967.

Preservation: Dry.

Remarks: Former Jens Hemmen Collection. Data on the specimen's label correspond to the lot originally catalogued as SMRL 3471. The specimen listed above is a possible remainder of a 30-specimen paratype lot left under the care of the SMRL. 


\section{triteia, Seguenzia Salvador, Cavallari \& Simone, 2014}

Seguenzia triteia Salvador, Cavallari \& Simone, 2014b: 548 (figs 25-28).

Gastropoda, Seguenziidae

Paratypes (3 spc): MZSP 116280.

Locality: Brazil, Espírito Santo, off São Mateus, Abrolhos Slope, 1859' S, 37²4' W, MD55 sta. DC70, 1540-1550 m depth, 26 May 1987.

Collectors: P. Bouchet, J.H. Leal and B. Métivier.

Preservation: Dry.

Remarks: Former MNHN, Paris.

\section{tupinamba, Calliostoma Dornellas, 2012}

Calliostoma tupinamba Dornellas, 2012: 91-101 (figs 1-9, 16-28).

Gastropoda, Calliostomatidae

Holotype: MZSP 105740.

Paratypes $^{1}$ (4 spc): MZSP 91745.

Paratypes $^{2}$ (7 spc): MZSP 102223.

Paratypes $^{3}$ (2 spc): MZSP 103766.

Paratype $^{4}$ : MZSP 105118.

Paratypes $^{5}$ (4 spc): MZSP 105660.

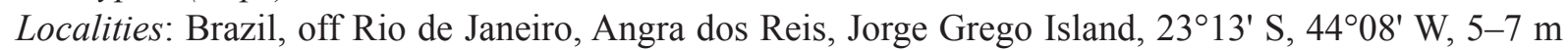
depth, 8-9 Aug. 2009; 1) from type locality; 2) off São Paulo, Alcatrazes Archipelago, Farol Island,

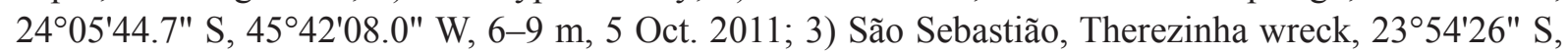

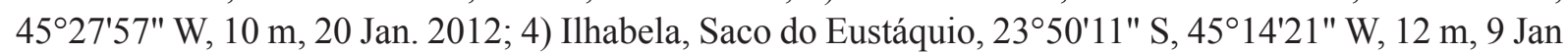
2012; 5) Vitória Island, Saco do Paiá, 234․ 658' S, 4501.343' W, 6-9 m, 15 May 2012.

Collector: A.P.S. Dornellas.

Preservation: $70 \%$ ethanol.

\section{umberlee, Halystina Salvador, Cavallari \& Simone, 2014}

Halystina umberlee Salvador, Cavallari \& Simone, 2014b: 542-544 (figs 13-18).

Gastropoda, Seguenziidae

Paratypes (12 spc): MZSP 116292.

Locality: Brazil, Espírito Santo, off São Mateus, Abrolhos Slope, 1859' S, 37²47' W, 1540-1550 m depth, 26 May 1987.

Collectors: P. Bouchet, J.H. Leal and B. Métivier.

Preservation: Dry.

Remarks: Former MNHN, Paris.

\section{unicolor, Andiniella flammulata Nordsieck, 2005}

Andiniella flammulata unicolor Nordsieck, 2005b: 212 (fig. 19).

Gastropoda, Clausiliidae

Paratype: MZSP 95490 (Fig. 5M-N).

Locality: Peru, Junin Department, end of Palca town to Tarma, left side of the road, $11^{\circ} 20.762^{\prime}$ S, 75³4.482' W (type locality), on rocks and under grass, 5 Apr. 2012.

Preservation: Dry.

Remarks: Former Jens Hemmen Collection. The specimen listed above is a remainder of a 20 -specimen paratype lot deposited by Nordsieck in Hemmen's collection. 
vlceki, Plekocheilus Breure \& Schlögl, 2010

Plekocheilus (Plekocheilus) vlceki Breure \& Schlögl, 2010: 52-54 (figs 2A-C, 3A, 3B, 4A).

Gastropoda, Orthalicidae

Paratype: MZSP 92470.

Locality: Venezuela, Bolivar State, Chimantá Massif, Churí-tepui, Sima Northwest, $2100 \mathrm{~m}$ altitude (type locality), Feb. 2009.

Collector: J. Schlögl.

Preservation: Dry.

watsoni, Aclis Barros, Lima \& Francisco, 2007

Aclis watsoni Barros, Lima \& Francisco, 2007: 65-67 (figs 7-9).

Gastropoda, Aclididae

Holotype: MZSP 77991.

Paratypes $^{1}$ (3 spc): MZSP 77992.

Paratypes $^{2}$ (4 spc): MZSP 77993.

Localities: Brazil, off Sergipe, continental slope, $10^{\circ} 41.4^{\prime} 00^{\prime \prime} \mathrm{S}, 36^{\circ} 18.7^{\prime} 00^{\prime \prime} \mathrm{W}, 365 \mathrm{~m}$ depth; 1) muddy

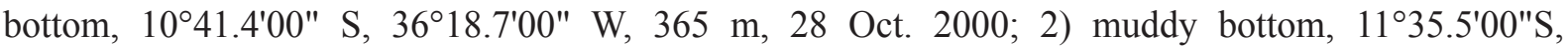
$37^{\circ} 12.3^{\prime} 00^{\prime \prime} \mathrm{W}, 510 \mathrm{~m}, 30$ Oct. 2000.

Preservation: Dry.

\section{wilhelminae, Diplommatina Maassen, 2002}

Diplommatina wilhelminae Maassen, 2002: 175 (figs 29-30).

Gastropoda, Diplommatinidae

Probable paratype: MZSP 95462 (Fig. 1K-L).

Locality: Indonesia, West Sumatra, SE of Bukit Tingii, $3 \mathrm{~km}$ E of Lintau, Cave Gua Pangian, $00^{\circ} 28^{\prime} 19.5^{\prime \prime} \mathrm{S}$, $100^{\circ} 45^{\prime} 11.7$ "E (type locality).

Preservation: Dry.

Remarks: Former Jens Hemmen Collection. Data on the specimen's label correspond to several paratype lots deposited in different collections, some of them private (Kittel Wiesthal, 2 specimens and Maassen's reference collection, 10 specimens).

\section{willianseverii, Heliacus Tenório, Barros, Francisco \& Silva, 2011}

Heliacus willianseverii Tenório, Barros, Francisco \& Silva, 2011: 186-189 (figs 5A-C, E-G).

Gastropoda, Architectonicidae

Paratype: MZSP 85949.

Paratype $^{1}$ : MZSP 85950.

Localities: Brazil, off Pernambuco, $09^{\circ} 07^{\prime} \mathrm{S}, 34^{\circ} 53^{\prime} \mathrm{W}$, D-Sinuelo, bottom with calcareous melobesia algae, 104 m (type locality), 17 Sep. 1999; 1) off Sergipe, 11 $58.7^{\prime}$ S, 36 $49.2^{\prime}$ W, D-Sinuelo, muddy bottom, 100 m, 1 Nov. 2000.

Preservation: Dry.

Remarks: Paratype MZSP 85949 corresponds to paratypes 8,9,10,11 and 12 from the original description by Tenório et al. (2011: 186). Similarly, MZSP 85950 corresponds to paratypes 13, 14, 15, 16 and 17. The authors gave incorrect MZSP catalogue numbers in the original description (from MZSP 15043 to MZSP 15052). These are actually non-type lots belonging to different species/mollusc groups registered in the 1980's, and must be disregarded. The valid numbers are the ones listed above. 


\section{Corrigendum to the previous list}

\section{inaequistriata, Helicina Pilsbry, 1900}

Helicina inaequistriata Pilsbry, 1900: 394.

Gastropoda, Helicinidae

Paralectotypes (17 spc): MZSP 938.

Locality: See Dornellas \& Simone (2011).

Collector: See Dornellas \& Simone (2011).

Preservation: Dry.

Remarks: The present specimens, also listed in Dornellas \& Simone (2011: 19), are actually paralectotypes. The lectotype and remaining paralectotypes are held in the Academy of Natural Sciences, Philadelphia (ANSP) collection, respectively catalogued as ANSP 78038 and ANSP 358655 (5 shells).

\section{Acknowledgements}

We are thankful to Vanessa S. Amaral (MZSP), Carlo M. Cunha (ANSP), Rodrigo B. Salvador (SMNS, Stuttgart) and Guido T. Poppe for valuable information and many helpful suggestions. We especially thank Daniel Abbate (MZSP) for providing several of the type photos and José and Marcus Coltro (Femorale) for providing several of the type specimens listed in this paper.

\section{References}

Absalão R.S. \& Oliveira C.D.C. 2011. The genus Cuspidaria (Pelecypoda: Septibranchia: Cuspidariidae) from the deep sea of Campos Basin, Brazil, with descriptions of two new species. Malacologia 54 (1-2): 119-138. http://dx.doi.org/10.4002/040.054.0104

Alf A. \& Kreipl K. 2006. A new Astraea from Bali, Indonesia. Spixiana 29 (1): 91-93.

Alf A. \& Kreipl K. 2011. The family Turbinidae. Subfamilies Turbininae Rafinesque, 1815 and Prisogasterinae Hickman \& McLean, 1990. In: Poppe G.T. \& Groh K. (eds) A Conchological Iconography. Conchbooks, Hackenheim.

Amaral V.S. \& Simone L.R.L. 2014. Revision of genus Crassostrea (Bivalvia: Ostreidae) of Brazil. Journal of the Marine Biological Association of the United Kingdom 94 (4): 811-836. http://dx.doi. org $/ 10.1017 / \mathrm{S} 0025315414000058$

Barros J.C.N., Lima S.F. \& Francisco J.A. 2007. Two new species of Aclis (Mollusca: Gastropoda: Aclididae) from the continental slope of northeast Brazil. Zootaxa 1614: 61-68.

Barros J.C.N., Santos P.C.P. \& Francisco J.A. 2008. Comments of species of Solariella and Lamellitrochus (Trochidae, Solariellinae) from the continental slope of Northeast Brazil, with the description of a new species. Revista Brasileira de Engenharia de Pesca 3 (1): 6-29.

Beck H.H. 1837. Index molluscorum praesentis aevi Musei Princepis augustissimi Christiani Frederici. Fasciculus primus. Copenhagen.

Benaim N.P. \& Absalão R.S. 2011. Deep sea Yoldiella (Pelecypoda: Protobranchia: Yoldiidae) from Campos Basin, Rio de Janeiro, Brazil. Journal of the Marine Biological Association of the United Kingdom 91 (2): 513-529. http://dx.doi.org/10.1017/S0025315410001372

Beu A.G. 2011. Marine Mollusca of isotope stages of the last 2 million years in New Zealand. Part 3. Gastropoda (Vetigastropoda - Littorinimorpha). Journal of the Royal Society of New Zealand 40 (3-4): 59-180. http://dx.doi.org/10.1080/03036758.2010.500717 
Blackwood T.J. 2009. Barneystrombus, a new genus of Strombidae (Gastropoda) from the Indo-West Pacific, with discussions of included taxa and the general morphology of their shells. Visaya 2 (5): $11-17$.

Bouchet P., Rocroi J.P., Frýda J., Hausdorf B., Ponder W.F., Valdés A. \& Warén A. 2005. Classification and nomenclator of gastropod families. Malacologia 47 (1-2): 1-397.

Brakoniecki T.F. 1984. A full description of Loligo sanpaulensis Brakoniecki, 1984 and a redescription of Loligo gahi d'Orbigny, 1835, two species of squid (Cephalopoda; Myopsida) from the southwest Atlantic. Bulletin of Marine Science 34 (3): 435-448.

Brandt R.A.M. 1959. Die Helicellinae der Cyrenaika. Archiv für Molluskenkunde 88 (4-6): 81-150.

Brandt R.A.M. 1968. Description of new non-marine mollusks from Asia. Archiv für Molluskenkunde 98 (5-6): 213-289.

Brandt R.A.M. 1970. New freshwater gastropods from Mekong. Archiv für Molluskenkunde 100 (3-4): 183-205.

Brandt R.A.M. 1974. The non-marine aquatic Mollusca of Thailand. Archiv für Molluskenkunde 105: $1-423$.

Breure A.S.H. \& Schlögl J. 2010. Additional notes on Orthalicidae from Chimantá massif, Venelzuelan Guayana, with descriptions of new species of Plekocheilus Guilding, 1828 (Mollusca: Gastropoda). Zootaxa 2416: 51-60.

Cavallari D.C., Salvador R.B. \& Cunha B.R. 2014. Dangers to malacological collections: Bynesian decay and pyrite decay. Collection Forum 28 (1-2): 35-46. http://dx.doi.org/10.14351/0831-0005$\underline{28.1 .35}$

Coltro J. \& Dornellas A.P.S. 2013. A new species of Anna (Mollusca: Neogastropoda: Buccinidae) from Brazil. Zoologia 30 (1): 97-100. http://dx.doi.org/10.1590/S1984-46702013000100012

Costa F.H.A. 1983. Anachis helenae, nova espécie de Columbellidae do litoral brasileiro (Mollusca: Gastropoda). Anais da Sociedade Nordestina de Zoologia 1 (1): 95-99.

Costa P.M.S. \& Simone L.R.L. 2006. A new species of Lucapina (Vetigastropoda, Fissurellidae) from Canopus Bank, N.E. Brazil. Strombus 13 (1): 1-5.

Costa P.M.S. \& Pimenta A.D. 2012. Revision of the genus Pazinotus (Gastropoda, Muricidae) from Brazil. American Malacological Bulletin 30 (1): 117-126. http://dx.doi.org/10.4003/006.030.0109

Cunha C.M. 2011. A new species of Acteon (Opisthobranchia: Acteonidae) from Northeast Brazil. Zoologia 28 (2): 229-232. http://dx.doi.org/10.1590/S1984-46702011000200012

Dacosta S., Cunha C.M., Simone L.R.L. \& Schrödl M. 2007. Computer-based 3-dimensional reconstruction of major organ systems of a new aeolid nudibranch subspecies, Flabellina engeli lucianae, from Brazil (Gastropoda: Opisthobranchia). Journal of Molluscan Studies 73: 339-353. http://dx.doi. org/10.1093/mollus/eym035

Davis G. 1979. The Origin and Evolution of the Gastropod Family Pomatiopsidae, with Emphasis on the Mekong River Triculinae. The Academy of Natural Sciences of Philadelphia, Allen Press Inc., Kansas.

Davis G., Subba Rao N.V. \& Hoagland K.E. 1986. In search of Tricula (Gastropoda: Prosobranchia): Tricula defined, and a new genus described. Proceedings of the Academy of Natural Sciences of Philadelphia 138 (2): 426-442.

Domínguez M., García F.J. \& Troncoso J.S. 2006. Anew species of Hoplodoris Bergh, 1880 (Gastropoda: Opisthobranchia: Nudibranchia) from the Atlantic Ocean. The Nautilus 120 (4): 150-155. 
Dornellas A.P.S. 2012. Description of a new species of Calliostoma (Gastropoda, Calliostomatidae) from Southeastern Brazil. ZooKeys 224: 89-106. http://dx.doi.org/10.3897/zookeys.224.3684

Dornellas A.P.S. \& Simone L.R.L. 2011. Annotated list of type specimens of mollusks deposited in Museu de Zoologia da Universidade de São Paulo, Brazil. Arquivos de Zoologia 42 (1): 1-81. http:// dx.doi.org/10.11606/issn.2176-7793.v42i1p1-81

Fernandes M.R., Pimenta A.D. \& Leal J.H. 2013. Taxonomic review of Triphorinae (Gastropoda: Triphoridae) from the Vitória-Trindade Seamount Chain, southeastern Brazil. The Nautilus 127 (1): $1-18$.

Figueira R.M.A. \& Absalão R.S. 2010a. Deep-water Mangeliinae, Taraninae and Clathurellinae (Mollusca: Gastropoda: Comoidea: Turridae) from the Campos Basin, southeast Brazil. Scientia Marina 74 (4): 731-743. http://dx.doi.org/10.3989/scimar.2010.74n4731

Figueira R.M.A. \& Absalão R.S. 2010b. Deep-water Drilliinae, Cochlespirinae and Oenopotinae (Mollusca: Gastropoda: Turridae) from the Campos Basin, southeast Brazil. Scientia Marina 74 (3): 471-481. http://dx.doi.org/10.3989/scimar.2010.74n3471

Francisco J.A.N., Barros J.C.N. \& Lima S.F. 2012. Five new species of Arcidae from Brazil with description of new genus: Paranadara (Mollusca: Bivalvia). Journal of the Marine Biological Association of the United Kingdom 92 (5): 1139-1150. http://dx.doi.org/10.1017/S0025315411000919

Gittenberger E. \& Hausdorf B. 2004. The Orculella species of the South Aegean Island arc, a neglected radiation (Gastropoda, Pulmonata, Orculidae). Basteria 68 (4-6): 93-124.

Haimovici M. 1988. Eledone gaucha, a new species of eledonid octopod (Cephalopoda: Octopodidae) from southern Brazil. The Nautilus 102 (2): 82-87. http://dx.doi.org/10.5962/bhl.part.5184

Ihering H. 1907. Les mollusques fossiles du Tertiaire et du Crétacé Supérieur de l'Argentine. Anales del Museo Nacional de Buenos Aires (3) 7: 1-611.

Jardim J.A. \& Simone L.R.L. 2010a. Redescription of Hanleya brachyplax (Polyplacophora, Hanleyidae) from the south-southeastern Brazilian coast. Papéis Avulsos de Zoologia 50 (40): 623-633.

Jardim J.A. \& Simone L.R.L. 2010b. Corrigenda for the paper "Redescription of Hanleya brachyplax (Polyplacophora, Hanleyidae) from the south-southeastern Brazilian coast" by Jardim \& Simone (2010). Strombus 17 (1-2): 14-15.

Jardim J.A., Abbate D. \& Simone L.R.L. 2013. A new species of Euglandina (Pulmonata, Spiraxidae) from Brazil. Journal of Conchology 41 (3): 327-330.

Kittel K., Groh K. \& Bank R.A. 2012. Nachruf auf Jens Hemmen * 9.1.1944 - †20.4.2012. Mitteilungen der Deutschen Malakozoologischen Gesellschaft 87: 51-66.

Lima S.F.B., Barros J.C.N. \& Petit R.E. 2007a. A new species of Gerdiela (Gastropoda: Cancellariidae) from the South Atlantic Ocean off Brazil with discussion of an undescribed species. The Nautilus 121 (2): 99-103.

Lima S.F.B., Tenório D.O. \& Barros J.C.N. 2007b. New species of Brazilian deep-water Terebra (Caenogastropoda: Terebridae) with first record of Terebra colombiensis Simone \& Gracia, 2006 from the southwestern Atlantic. Miscellanea Malacologica 2 (4): 63-72.

Lima S.F.B., Barros J.C.N. \& Francisco J.A. 2010. A new deep-sea species of Mitromorpha (Gastropoda: Conoidea: Conidae) off Brazil. Journal of Marine Biological Association of the United Kingdom 90 (3): 599-603. http://dx.doi.org/10.1017/S0025315409990762 
Lima S.F.B., Santos F.N. \& Absalão R.S. 2013. New species of Caecum (Caenogastropoda: Rissooidea: Caecidae) from the Atlantic Coast of South America (Brazil) with a description of the protoconch and growth stages. Zoological Science 30 (9): 767-778. http://dx.doi.org/10.2108/zsj.30.767

Lyons W.G. \& Snyder M.A. 2013. The genus Pustulatirus Vermeij and Snyder, 2006 (Gastropoda: Fasciolariidae: Peristerniinae) in the Western Atlantic, with descriptions of three new species. Zootaxa 3636 (1): 35-58. http://dx.doi.org/10.11646/zootaxa.3636.1.2

Maassen W.J.M. 2000. Notes on terrestrial molluscs of Sumatra, Indonesia, with descriptions of ten new species (Gastropoda, Prosobranchia \& Pulmonata). Basteria 64 (4-6): 137-150.

Maassen W.J.M. 2001. Four new Diplommatinidae (Gastropoda, Prosobranchia, Diplommatinidae) from southern Thailand and northern Peninsular Malaysia. Basteria 65 (1-3): 51-56.

Maassen W.J.M. 2002. Remarks on the Diplommatinidae from Sumatra, Indonesia, with descriptions of eleven new species (Gastropoda, Prosobranchia). Basteria 66 (4-6): 163-182.

Maassen W.J.M. 2007. Notes on terrestrial molluscs of the island of Sulawesi. 4. The genus Diplommatina (Gastropoda, Caenogastropoda, Diplommatinidae). Basteria 71: 189-208.

Mansur M.C.D. \& Pimpão D.M. 2008. Triplodon chodo, a new species of pearly fresh water mussel from the Amazon Basin (Mollusca: Bivalvia: Unioidea: Hyriidae). Revista Brasileira de Zoologia 25 (1): 111-115. http://dx.doi.org/10.1590/S0101-81752008000100015

Marques R.C. \& Simone L.R.L. 2011. A new species of Ervilia from North Brazil (Bivalvia, Semelidae). Journal of Conchology 40 (6): 651-655.

Marques R.C. \& Simone L.R.L. 2014. Eurytellina angrensis, a new species from southeastern Brazilian coast, with anatomical details. Archiv für Molluskenkunde 143 (1): 39-50. http://dx.doi.org/10.1127/ arch.moll/1869-0963/143/039-050

Mühlhäusser H. 1981. Strombus kleckhamae n. subsp. Spixiana 4 (3): 319-324.

Neubert E. \& Groh K. 1998. Contributions to the nomenclature and phylogeny of Boettgeria O. Boettger, 1863, with description of Loosjesiella n. subgen. (Gastropoda: Pulmonata: Clausiliidae). Basteria 62 (3-4): 157-168.

Nordsieck H. 2005a. New Clausiliidae from China and Taiwan. Archiv für Molluskenkunde 134 (1): $23-52$.

Nordsieck H. 2005b. Revision of the system of the Peruvian Neniinae, with description of new taxa. Archiv für Molluskenkunde 134 (2): 197-221. http://dx.doi.org/10.1127/arch.moll/0003-9284/134/197$\underline{221}$

Nordsieck H. 2010. New taxa of the subfamilies Neniinae and Garnieriinae (Gastropoda: Stylommatophora: Clausiliidae). Archiv für Molluskenkunde 139 (1): 45-69. http://dx.doi.org/10.1127/ arch.moll/1869-0963/139/045-069

Padula V., Araújo A.K., Matthews-Cascon H. \& Schrödl M. 2014. Is the Mediterranean nudibranch Cratena peregrina (Gmelin, 1791) present on the Brazilian coast? Integrative species delimitation and description of Cratena minor n. sp. Journal of Molluscan Studies 80: 575-584. http://dx.doi.org/10.1093/ mollus/eyu052

Passos F.D. \& Machado F.M. 2014. A new species of Cyamiocardium Soot-Ryen, 1951 from shallow waters off Brazil, with a discussion on the anatomical characters of the Cyamiidae (Bivalvia: Cyamioidea). American Malacological Bulletin 32 (1): 122-131. http://dx.doi.org/10.4003/006.032.0110

Perez J.A.A. \& Haimovici M. 1991. Cephalopod collection of 'Museu de Zoologia da Universidade de Sao Paulo', Sao Paulo, Brazil. Papéis Avulsos de Zoologia 37 (16): 251-258. 
Petuch E.J. \& Myers R.F. 2014a. New species of Conidae and Conilithidae (Gastropoda: Conoidea) from the Bahamas, Eastern Caribbean, and Brazil. Xenophora Taxonomy 3: 34-47.

Petuch E.J. \& Myers R.F. 2014b. Additions to the cone shell faunas (Conidae and Conilithidae) of the Cearaian and Bahian Subprovinces, Brazilian Molluscan Province. Xenophora Taxonomy 4: 30-43.

Pilsbry H.A. 1900. New South American land snails. Proceedings of the Academy of Natural Sciences of Philadelphia 52: 385-394.

Pimenta A.D., Santos F.N. \& Absalão R.S. 2008. Review of the genera Ividia, Folinella, Oscilla, Pseudoscilla, Tryptichus and Peristichia (Gastropoda, Pyramidellidae) from Brazil, with description of four new species. The Veliger 50 (3): 171-184.

Pimenta A.D., Absalão R.S. \& Miyaji C. 2009. A taxonomic review of the genera Boonea, Chrysallida, Parthenina, Ivara, Fargoa, Mumiola, Odostomella and Trabecula (Gastropoda, Pyramidellidae, Odostomiinae) from Brazil. Zootaxa 2049: 39-66.

Pimenta A.D., Santos F.N. \& Absalão R.S. 2011. Taxonomic revision of the genus Eulimella (Gastropoda, Pyramidellidae) from Brazil, with description of three new species. Zootaxa 3063: 22-38.

PimentaA.D. 2012. Four new species and two new records of Odostomiinae (Gastropoda: Pyramidellidae) from Brazil. Zoologia 29 (5): 439-450. http://dx.doi.org/10.1590/S1984-46702012000500007

Poppe G.T. \& Goto Y. 1993. Recent Angariidae. L'Informatore Piceno, Ancona, Italy.

Powell W.B. 1927. Mollusca from twenty-three fathoms off Ahipara, N.Z. Transactions of the New Zealand Institute 58: 295-300.

Puillandre N., Duda T.F., Meyer C., Olivera B.M. \& Bouchet P. 2015. One, four or 100 genera? A new classification of the cone snails. Journal of Molluscan Studies 81: 1-23. http://dx.doi.org/10.1093/ $\underline{\text { mollus/eyu055 }}$

Rios E.C. 2009. Compendium of Brazilian Seashells. Evangraf, Rio Grande.

Salvador R.B. \& Cavallari D.C. 2013a. A new Oxychona species (Gastropoda: Pulmonata: Orthalicidae) from Bahia state, Brazil. Journal of Conchology 41 (3): 315-318.

Salvador R.B. \& Cavallari D.C. 2013b. Taxonomic revision of Leiostracus onager and Leiostracus subtuszonatus (Gastropoda: Pulmonata: Orthalicidae). Journal of Conchology 41 (4): 511-518.

Salvador R.B. \& Cavallari D.C. 2014. A new species of Leiostracus from Bahia, Brazil (Gastropoda, Pulmonata, Orthalicidae). Iheringia, Série Zoologia 104 (1): 39-42.

Salvador R.B., Cavallari D.C. \& Breure A.S.H. 2014a. Corrigendum to "Taxonomic revision of Leiostracus onager and Leiostracus subtuszonatus (Gastropoda: Pulmonata: Orthalicidae)" by Salvador \& Cavallari (2013). Journal of Conchology 41 (5): 627-628.

Salvador R.B., Cavallari D.C. \& Simone L.R.L. 2014b. Seguenziidae (Gastropoda: Vetigastropoda) from SE Brazil collected by the Marion Dufresne (MD55) expedition. Zootaxa 3878 (6): 536-550. http://dx.doi.org/10.11646/zootaxa.3878.6.2

Simone L.R.L. 2009. Comparative morphology among representatives of main taxa of Scaphopoda and basal protobranch Bivalvia (Mollusca). Papéis Avulsos de Zoologia 49 (32): 405-457.

Simone L.R.L. 2011. Phylogeny of the Caenogastropoda (Mollusca), based on comparative morphology. Arquivos de Zoologia 42 (4):161-323. http://dx.doi.org/10.11606/issn.2176-7793.v42i4p161-323

Simone L.R.L. 2012a. A new genus and species of cavernicolous Pomatiopsidae (Mollusca, Caenogastropoda) in Bahia, Brazil. Papéis Avulsos de Zoologia 52 (40): 515-524. http://dx.doi. org/10.1590/S0031-10492012022000001 
Simone L.R.L. 2012b. Taxonomical study on a sample of pulmonates from Santa Maria da Vitória, Bahia, Brazil, with description of a new genus and four new species (Mollusca: Orthalicidae and Megalobulimidae). Papéis Avulsos de Zoologia 52 (36): 431-439.

Simone L.R.L. 2012c. Two new species of Tornidae (Caenogastropoda, Rissooidea) from Espírito Santo, Brazil. ZooKeys 238: 77-85. http://dx.doi.org/10.3897/zookeys.238.3884

Simone L.R.L. \& Cunha C.M. 2012. Taxonomic study on the molluscs collected in MarionDufresne expedition (MD55) to SE Brazil; Xenophoridae, Cypraeoidea, mitriforms and Terebridae (Caenogastropoda). Zoosystema 34 (4): 745-781. http://dx.doi.org/10.5252/z2012n4a6

Simone L.R.L. 2013a. A new Brazilian tornid is possibly the flattest coiled snail. Journal of Molluscan Studies 79: 187-189. http://dx.doi.org/10.1093/mollus/eyt004

Simone L.R.L. 2013b. Habeas, a new genus of Diplommatinidae from central Bahia, Brazil (Caenogastropoda), with description of three new species. Journal of Conchology 41 (4): 519-525.

Simone L.R.L. \& Casati R. 2013. New land mollusk fauna from Serra da Capivara, Piauí, Brazil, with a new genus and five new species (Gastropoda: Orthalicoidea, Streptaxidae, Subulinidae). Zootaxa 3683 (2): 145-158. http://dx.doi.org/10.11646/zootaxa.3683.2.4

Simone L.R.L. 2014. Taxonomic study on the molluscs collected during the Marion-Dufresne expedition (MD55) off SE Brazil: the Naticidae (Mollusca: Caenogastropoda). Zoosystema 36 (3): 563-593. http:// dx.doi.org/10.5252/z2014n3a2

Simone L.R.L. \& Cunha C.M. 2014. Taxonomical study on the mollusks collected in Marion-Dufresne (MD55) and other expeditions to SE Brazil: the Fissurellidae (Mollusca, Vetigastropoda). Zootaxa 3835 (4): 437-468. http://dx.doi.org/10.11646/zootaxa.3835.4.2

Souza P.S. de \& Coovert G.A. 2001. Revision of the recent Bullata Jousseaume, 1875 (Gastropoda: Marginellidae) with the description of two new species. The Nautilus 115 (1): 1-14. http://dx.doi. org/10.5962/bhl.part.11263

Spotorno P. \& Simone L.R.L. 2013. First record of Thylaeodus (Gastropoda: Vermetidae) from the Equatorial Atlantic Ocean, with the description of a new species. Zoologia 30 (1): 88-96. http://dx.doi. org/10.1590/S1984-46702013000100011

Subai P. 2005. Revision der Gattung Codringtonia Kobelt 1898 (Gastropoda: Pulmonata: Helicidae: Helicinae). Archiv für Molluskenkunde 134 (1): 65-119. http://dx.doi.org/10.1127/arch.moll/0003$\underline{9284 / 134 / 065-119}$

Tavares M. 1999. The cruise of the Marion Dufresne off the Brazilian coast: account of the scientific results and list of stations. Zoosystema 21 (4): 597-605.

Temcharoen P. 1971. New aquatic molluscs from Laos. Archiv für Molluskenkunde 101 (1): 91-109.

Tenório D.O., Barros J.C.N., Francisco J.A. \& Silva G.F. 2011. New species of Architectonicidae (Gastropoda Heterobranchia) from northeastern Brazil. Tropical Zoology 24: 173-191.

Vecchione M., Brakoniecki T.F., Natsukari Y. \& Hanlon R.T. 1998. A provisional generic classification of the family Loliginidae. In: Voss N.A., Vecchione M., Toll R.B. \& Sweeney M.J. (eds) Systematics and Biogeography of Cephalopods. Vol. 1: 215-222. Smithsonian Contributions to Zoology 586, Smithsonian Institution Press, Washington, DC.

WoRMS. 2015. Mollusca [online]. Available from: http://www.marinespecies.org/aphia. php?p=taxdetails\&id=51 [accessed 10 Nov. 2015] 
Manuscript received: 12 June 2015

Manuscript accepted: 20 February 2016

Published on: 18 July 2016

Topic editor: Rudy Jocqué

Section editor: Kurt Jordaens

Desk editor: Charlotte Thionois

Printed versions of all papers are also deposited in the libraries of the institutes that are members of the EJT consortium: Muséum national d'Histoire naturelle, Paris, France; Botanic Garden Meise, Belgium; Royal Museum for Central Africa, Tervuren, Belgium; Natural History Museum, London, United Kingdom; Royal Belgian Institute of Natural Sciences, Brussels, Belgium; Natural History Museum of Denmark, Copenhagen, Denmark; Naturalis Biodiversity Center, Leiden, the Netherlands. 